\title{
Maintenance in Railway Rolling Stock Rescheduling for Passenger Railways
}

\author{
Joris Wagenaar and Leo Kroon \\ Rotterdam School of Management, Erasmus University \\ Technology and Operations Management \\ Burgemeester Oudlaan 50, 3062 PA, Rotterdam The Netherlands \\ e-mail: jwagenaar@rsm.nl, lkroon@rsm.nl
}

February 18, 2015

\begin{abstract}
This paper addresses the Rolling Stock Rescheduling Problem (RSRP), while taking maintenance appointments into account. After a disruption, the rolling stock of passenger trains has to be rescheduled in order to maintain a feasible rolling stock circulation. A limited number of rolling stock units have a scheduled maintenance appointment during the day: these appointments need to be taken into account while rescheduling. In this paper we propose three different models for this. The Extra Unit Type model extends the known Composition model by adding additional rolling stock types for every rolling stock unit that requires maintenance. The Shadow-Account model keeps track of a shadow account for all units that require maintenance. The Job-Composition model is a combination of the Job model and the Composition model, both known in the literature. Paths are created such that maintenance units are on time for their maintenance appointment. All models are tested on instances of Netherlands Railways. The results show that the models are able to efficiently take maintenance appointments into account.
\end{abstract}

\section{Introduction}

In passenger railway transportation, an extensive planning period is used to develop a satisfying rolling stock circulation. During this planning period, maintenance required by the rolling stock can be scheduled by adjusting the rolling stock schedule such that the maintenance appointments are met by the correct rolling stock unit arriving at the right station on time, see for instance Maróti and Kroon [9]. Throughout the daily operations, the railway network inevitably experiences disruptions and therefore fast rescheduling 
is required. The Rolling Stock Rescheduling Problem (RSRP) aims to find a new rolling stock schedule that upholds as much of the passenger service as possible and as swiftly as possible. However, during real-time operations there is not sufficient time available to (re)schedule maintenance appointments as done during the planning phase. As a consequence, it is desirable that maintenance appointments can be taken into account directly in the rescheduling process.

Rolling stock units of different types are available for passenger transportation. There exist large differences between the different types, there are for instance trains with two floors (called double-deck) and trains with a single floor. In this paper we only look at rolling stock types with small differences. The main difference between the different types we consider is the number of carriages of which they consist (e.g. a VIRM6 consists of 6 carriages and a VIRM4 of 4 carriages). See, for instance, Figure 1 for a rolling stock unit of type VIRM4.

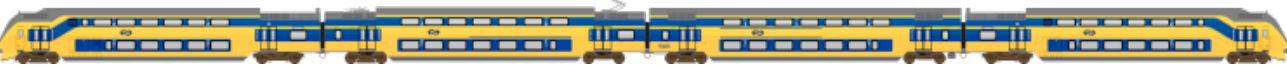

Figure 1: Rolling stock of type VIRM4

Current rolling stock rescheduling models, see for example Fioole et al. [6], assume that all rolling stock units of the same type are interchangeable. That means, for instance, that there is no distinction between units that require maintenance and units that do not. As a result, after rescheduling, the rolling stock units scheduled for maintenance will probably not be in time for their appointment. The main contribution of this paper is the development of three new models which are able to handle the complicating factor that units within the same type are no longer fully interchangeable.

In passenger railway transportation, rolling stock units can be coupled to each other to form a rolling stock composition. In this way more capacity can be appointed to a trip. The order of the different units in a train composition is of importance due to (un)coupling restrictions. The (un)coupling actions of rolling stock units in a composition depend on the positions of other units within the same composition (e.g. the unit in the middle of a composition of 3 units cannot be uncoupled). As a result, the usage of, for instance, a column generation technique is not evident. Therefore, a Mixed Integer Programming (MIP) formulation for solving additonally constrained MultiCommodity Flow Problems is used as solution method for all three models.

The paper begins with a literature overview in Section 2. Then, the Composition model is presented. This model is used as base model for all three models that take maintenance into account. Thereafter, in Section 4, the maintenance problem is explained in detail. Following, three different approaches for including maintenance in rolling stock rescheduling models are given. First, the Extra Unit Type model is discussed in Section 5. 
Secondly, the Shadow-Account model is presented in Section 6, and finally the Job-Composition model is proposed in Section 7. Then, in Section 8, all models are tested on real life instances of Netherlands Railways (NS) and compared with respect to their computation time.

\section{Literature}

Cacchiani et al. [4] give an extensive literature overview on recent research within passenger railway disruption management. Papers on rescheduling the timetable on microscopic and macroscopic level, rescheduling the rolling stock, and rescheduling the crew are discussed. We refer to this paper for all literature on timetable and crew rescheduling. In the current paper the focus is on rescheduling the rolling stock, so the remainder of the discussed literature will be on rolling stock.

Fioole et al. [6] formulate a MIP model to assign rolling stock to the timetable. The model is called the Composition model and is a MultiCommodity Flow Model with additional constraints. It is able to handle complicated line structures, such as combining and splitting of trains. NS has been using this model to generate the rolling stock schedules since 2004 . Their model does take the order of train units specifically into account, however, maintenance routing is out of their scope.

The routing of maintenance units is a well studied problem in the aircraft industry. For instance, Barnhart et al. [2], Talluri [15], and Clarke et al. [5] propose models to solve this problem. Their models cannot be directly translated to railway scheduling models maintenance scheduling due to practical complications, such as the order of train units within a composition.

For this reason, Maróti and Kroon [9] and Maróti and Kroon [10] propose two different MIP formulations for maintenance routing of rolling stock in the passenger railway industry: the "Transition Model" and the "Interchange Model". Both models take the scheduled rolling stock circulation as input into account, and exchange unit duties such that maintenance requirements are met.

Borndörfer et al. [3] introduce a hypergraph formulation to create a rolling stock circulation for a generic week. In this circulation several important practical requirements are taken into account. One of these requirements is the scheduling of maintenance for rolling stock units. Their model is tested on real life instances of Deutsche Bahnh. Circulations are found in between 10 minutes and 4 days of computation time.

All of the above models are only applicable in the planning phase of the railway process. During a disruption less time is available, and as a result fast models have to be used to reschedule the rolling stock. Nielsen [11] extends the model of Fioole et al. [6] to cope with rescheduling. He formulates a MIP model with the adjusted timetable and the original rolling 
stock schedule as input, and an adjusted rolling stock schedule as output. This model will be used as base model in this paper and is referred to as the Composition model.

Subsequently, Nielsen et al. [12] propose a rolling horizon to solve the RSRP. The idea behind the rolling horizon is that at the beginning of the disruption not all information about the duration of the disruption is known: this information becomes gradually available. The rescheduling is periodically performed within a limited rolling horizon length, possibly taking new information into account. At each time instant where an updated timetable becomes available, or when a certain amount of time has passed without any update, the MIP model is solved for the rolling horizon time window. This model is tested on instances of NS. Solutions with small deviations from the original plan are found in a short time.

Kroon et al. [7] consider real-time rescheduling of rolling stock during large disruptions while taking dynamic passenger flows into account. They use a two-stage feedback loop, where in the first stage the rolling stock allocation is optimized by using the model of Nielsen [11] and in the second stage the effect of the rolling stock allocation on passenger flows is determined by means of simulation. This simulation provides feedback in terms of passenger delays due to limited capacity of the assigned rolling stock. The feedback is then used in the optimization model to reallocate the rolling stock again, in such a way that the total passenger delay is reduced. Given the reallocation of the rolling stock, the passenger simulation is performed again and feedback is given to the optimization model. This process continues for a number of iterations.

Sato et al. [14] give a formulation to reallocate resources in a railway network in case of a disruption. Resources may refer to either rolling stock or to crew. The resources are reallocated to trips in such a way that they differ as little as possible from the ones in the original plan. They use two phases to solve the problem: in the first phase conflicts created by the disruption are resolved through small changes in the resource duties. The second phase is a local search heuristic which attempts to iteratively improve the rescheduled resource duties. The algorithm is tested on one line of the Japanese railway network.

In a subsequent paper, Sato and Fukumura [13] consider the problem of reassigning locomotives to tasks in the case of a disruption in the railway network. A task consists of hauling a number of freight carriages from one station to another. They first enumerate possible sequences of tasks, to determine the corresponding costs for each sequence. A MIP model based on set-partitioning is used in order to assign locomotives to sequences of tasks with minimum cost, and a column generation technique is proposed as a solution approach. Based on the solutions found for instances of the Japan Freight Railway Company between Kuroiso and Shimonoseki in Japan, the authors conclude that locomotive reassignments can be found within a prac- 
tical amount of time.

\section{Composition model}

We start with introducing the base model, this model is based on the Composition model created by Fioole et al. [6] and Nielsen [11].

Let $T$ be the set of trips in the timetable and $S$ the set of stations. A trip is defined as a train driving from one station where the composition may be changed until the next station where the composition may be changed again at a fixed point in time. Denote $s_{t}^{d e p}\left(s_{t}^{\text {arr }}\right)$ as the station where trip $t \in T$ starts (ends) and define $\tau_{t}^{d e p}\left(\tau_{t}^{a r r}\right)$ as the departure (arrival) time of trip $t \in T$.

In most countries trips are part of a predefined route. By this we mean that a trip either has a predefined successor trip, or that the route ends after the trip. Take Figure 2 as an example of a predefined route between stations $\mathrm{A}, \mathrm{B}$, and $\mathrm{C}$. There are two trips between stations $\mathrm{A}$ and $\mathrm{B}$, two trips between stations $\mathrm{B}$ and $\mathrm{C}$, two trips between stations $\mathrm{C}$ and $\mathrm{B}$, and two trips between stations $\mathrm{B}$ and $\mathrm{A}$. Trip $t_{1}$ is the first trip of the route, so $t_{1}$ is not a successor of any other trip. Thereafter, we have that trip $t_{2}$ succeeds $t_{1}, t_{3}$ succeeds $t_{2}$, and so on. Trip $t_{8}$ does not have a successor, so the route ends after trip $t_{8}$. If no coupling or uncoupling takes place, it means that the successor trip can make use of exactly the same rolling stock units as its predecessor. If a trip does not have a successor, so the route ends, it means that all rolling stock units are moved to the shunting yard.

As can be seen in Figure 2, also trips at the end of a line can have a successor. For instance, trip 3 succeeds trip 2. This is because turning can take place between two successor trips. In that case, the rolling stock units that will be used on the successor trip, wait at the track where the trip arrives until the successor trip departs.

We define $\sigma(t)$ as the successor trip of trip $t, R$ as the set of routes, and $r:=\left(t_{1}, \cdots, t_{n}\right)$ is a route consisting of a sequence of trips, such that $t_{1}$ does not have a predecessor, that $\sigma\left(t_{i}\right)=t_{i+1}$ for all $i=1, \cdots, n-1$ and that $\sigma\left(t_{n}\right)=\emptyset$. Then, $r(t)$ is the (uniquely defined) route trip $t$ is assigned to.

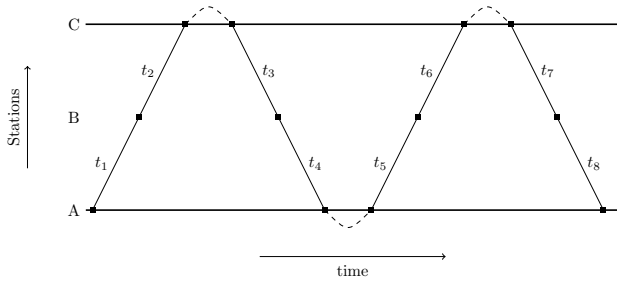

Figure 2: Predefined route

Now, let $M$ be the set of rolling stock types. We denote $P$ as the set 
of possible compositions, where a composition is an ordered combination of rolling stock units that can be used on a trip. For example, in Figure 3 the composition $a b$ is appointed to trip $t$ and the composition $a$ is appointed to trip $\sigma(t)$. For each trip $t \in T, P(t)$ denotes the set of allowed compositions on the trip. Note that the empty composition is within $P(t)$ for each trip, meaning that the trip is cancelled.

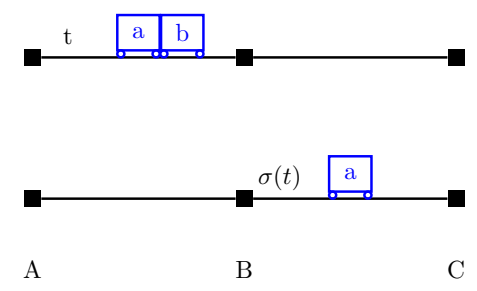

Figure 3: The successor of trip $t$

At the end of a trip, the composition of a train can possibly be changed, depending on the shunting rules at the station, before departing on its successor trip. A composition change denotes the composition of the incoming trip, the composition of the outgoing trip, and which units are coupled or uncoupled during the composition change. To that end, let $\rho(t)$ be the set of possible composition changes at the end of trip $t \in T, p_{q}$ the incoming composition of a trip when composition change $q \in \rho(t)$ is used, and $o_{q}$ the outgoing composition when composition change $q$ is used. For a given composition change $q \in \rho(t), \alpha_{q, m}$ denotes the number of uncoupled units of type $m \in M$ and $\beta_{q, m}$ denotes the number of coupled units of type $m \in M$. For instance, the composition change $a b \rightarrow a$ takes place at station $\mathrm{B}$ in Figure 3, so $p_{q}=a b$ and $o_{q}=a$. Furthermore, $\alpha_{q, a}=0, \alpha_{q, b}=1$, and $\beta_{q, m}=0$ for both $m=a$ and $m=b$.

The time at which coupling takes place just before the start of trip $t \in T$ is denoted by $\tau_{t}^{+}$and the time at which an uncoupled unit is available after uncoupling after trip $t \in T$ is denoted by $\tau_{t}^{-}$. Coupling and uncoupling takes place at either the front or rear side of the train, this is defined by the station rules. Note that in the Netherlands it is not allowed to both couple and uncouple units at the end of the same trip. In the remainder of this paper that is assumed to be the case as well.

The available number of units of type $m \in M$ at station $s \in S$ at the beginning of the planning period is denoted by $i_{s, m}^{0}$ and the desired number of available units of type $m \in M$ at station $s \in S$ at the end of the planning period is given by the parameter $i_{s, m}^{\infty}$. This usually is the end of the day.

The following decision variables are used in the model:

- $X_{t, p} \in\{0,1\}$ denotes whether composition $p \in P(t)$ is used on trip $t \in T$. 
- $Z_{t, q} \in\{0,1\}$ denotes whether composition change $q \in \rho(t)$ is used at the end of trip $t \in T$.

- $I_{t, m} \in \mathbb{Z}_{0}^{+}$denotes the number of units of type $m \in M$ in the inventory at station $s_{t}^{\text {dep }}$ immediately after time $\tau_{t}^{+}$.

- $C_{t, m}$ and $U_{t, m} \in \mathbb{Z}_{0}^{+}$denote the number of units $m \in M$ that are coupled and uncoupled at the start and end of trip $t \in T$.

- $D_{s, m} \in \mathbb{Z}$ denotes the deviation from the desired end of day balance at station $s \in S$ for rolling stock type $m \in M$. 


\section{Model:}

$\min f(X, Z, D)$

subject to:

$\sum_{p \in P(t)} X_{t, p}=1$

$\forall t \in T$

$X_{t, p}=\sum_{q \in \rho(t): p_{q}=p} Z_{t, q}$

$\forall t \in T, p \in P(t)$

$X_{\sigma(t), p}=\sum_{q \in \rho(t): o_{q}=p} Z_{t, q}$

$C_{\sigma(t), m}=\sum_{q \in \rho(t)} \beta_{q, m} Z_{t, q}$

$U_{t, m}=\sum_{q \in \rho(t)} \alpha_{q, m} Z_{t, q}$

$\forall t \in T, m \in M$

$\forall t \in T, m \in M$

$I_{t, m}=i_{s_{t}^{d e p}, m}^{0}-\sum_{t^{\prime} \in A_{t}} C_{t^{\prime}, m}+\sum_{t^{\prime} \in B_{t}} U_{t^{\prime}, m}$

$\forall t \in T, m \in M$

$i_{s, m}^{\infty}=i_{s, m}^{0}-\sum_{t \in T, s_{t}^{d e p}=s} C_{t, m}+\sum_{t \in T, s_{t}^{a r r}=s} U_{t, m}+D_{s, m} \quad \forall s \in S, m \in M$

$X_{t, p} \in\{0,1\}$

$\forall t \in T, p \in P(t)$

$C_{t, m}, U_{t, m}, I_{t, m} \in \mathbb{R}_{+}$

$\forall t \in T, m \in M$

$D_{s, m} \in \mathbb{R}_{+}$

$\forall s \in S, m \in M$

$Z_{t, q} \in \mathbb{R}_{+}$

$\forall t \in T, q \in \rho(t)$

Here the subsets $A_{t}$ and $B_{t}$ are defined as:

1. $A_{t}=\left\{t^{\prime} \in T: s_{t^{\prime}}^{d e p}=s_{t}^{d e p}, \tau_{t^{\prime}}^{+} \leq \tau_{t}^{+}\right\}$

2. $B_{t}=\left\{t^{\prime} \in T: s_{t^{\prime}}^{a r r}=s_{t}^{d e p}, \tau_{t^{\prime}}^{-} \leq \tau_{t}^{+}\right\}$

Constraint (3.2) specifies that to each trip exactly one composition is assigned, this composition is in the set of allowed compositions, $P(t)$, of 
that trip. Note that the compositions of the trips before and at the start of the disruption are fixed, because these trips are already underway. For those trips the set of allowed compositions consists of only a single composition. Constraint (3.3) states that if composition $p \in P$ is assigned to trip $t \in T$, then only a composition that can originate from $p$ can be assigned to the succeeding trip $\sigma(t)$. Constraint (3.4) states that if composition $p \in P$ is assigned to the succeeding trip $\sigma(t)$, then only a composition that fits with composition $p$ can be assigned to trip $t \in T$.

Constraint (3.5) specifies the number of coupled rolling stock units at the beginning of a trip and Constraint (3.6) specifies the number of uncoupled rolling stock units at the end of a trip. Constraint (3.7) denotes the inventory level of rolling stock type $m \in M$ at station $s_{t}^{\text {dep }}$ immediately after time $\tau_{t}^{+}$. Note that at the shunting area there are no longer whole compositions present: all units within a composition are detached from each other at the shunting area, and as a result only the inventory per rolling stock type is registered. Constraint (3.8) specifies the end of day balance at a station plus the total deviation from the scheduled end of day balance.

The other constraints specify the character of the decision variables. Since $X_{t, p}$ is binary, all other variables can be defined as continuous variables. All constraints are defined such that those variables are integer in the solution, see Maróti [8].

The objective function (3.1) depends on the appointed compositions $(X)$, this includes the number of cancelled trips, the capacity shortages, and the number of carriage kilometers. The objective furthermore depends on the shunting movements $(Z)$ : modified shunting movements, with respect to the original plan, are penalized. Finally, the total deviation from the end of day balance $(D)$ is penalized.

The output of this model is a list of trips with compositions appointed to them. Note that these compositions can be decomposed into duties for rolling stock units, because an integer flow can always be decomposed into unit valued path flows, see Ahuja et al. [1]. However, the Composition model assumes all units of the same type $m \in M$ to be interchangeable. As a result, the model is unable to create a circulation where the rolling stock units that require maintenance are on time for their maintenance appointment.

\section{Maintenance problem}

In this section the maintenance problem is explained in detail. We start with an example of the problem, thereafter the notation used throughout the paper for the maintenance problem is discussed. 


\subsection{Example of the maintenance problem}

See Figure 4 for a time-space diagram of the scheduled rolling stock circulation on the 3000 series between the stations Nijmegen (Nm) and Den Helder (Hdr). This circulation is infeasible due to a disruption between the stations Utrecht (Ut) and Amsterdam (Asd) from 09:00-11:00. There are in total 25 rolling stock units available, whereof two rolling stock units require maintenance, one that starts in Alkmaar (Amr) with an appointment at 16:00 at station Nm (black line) and one that starts in Hdr with an appointment at 22:00 at station $\mathrm{Nm}$ (dark gray line). All units have an appointment that lasts for two hours, thereafter they are available for usage again. The circulation needs to be rescheduled for the remainder of the day, preferably such that the maintenance appointments are still reached by the corresponding rolling stock units which require it.

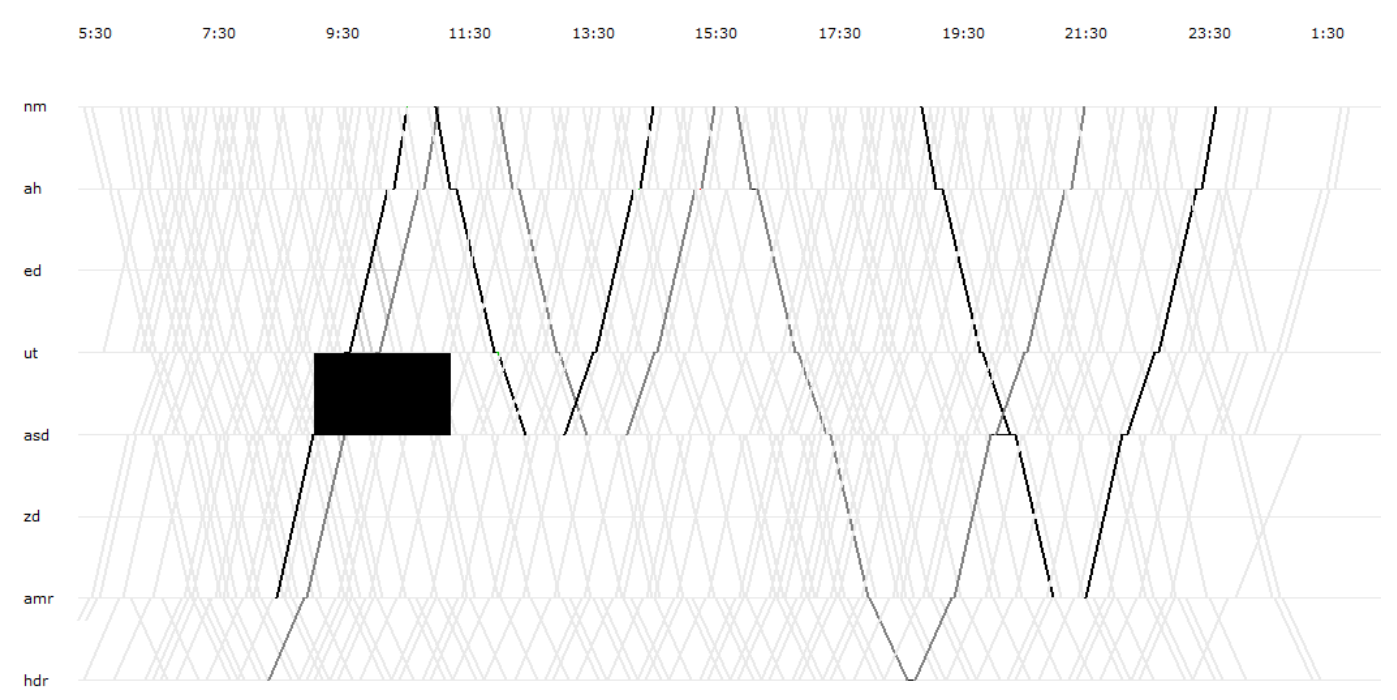

Figure 4: Time space diagram with a disruption

Figure 5 shows the solution after rescheduling. As can be seen, both units are still on time for their maintenance appointment. The models we discuss in the coming sections are able to reschedule the rolling stock in this way.

The problem is thus to guide individual rolling stock units in time to a maintenance facility. This requires extensions of the Composition model, since this model does not distinguish individual rolling stock units.

\subsection{Notation}

The notation for maintenance units we use throughout this paper is the following. Let $M^{\prime}$ be the set of rolling stock units that require maintenance. Denote $h_{m}$ as the time unit $m \in M^{\prime}$ has its maintenance appointment, $g_{m}$ as 


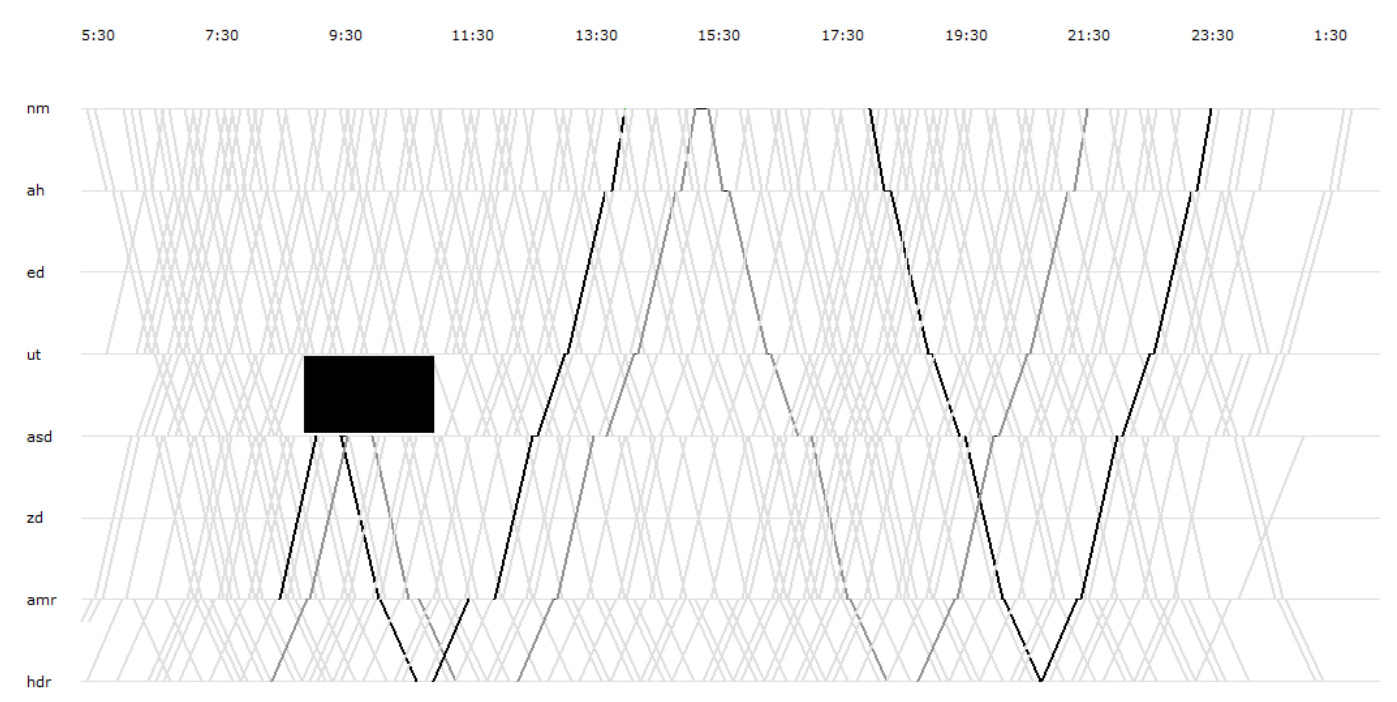

Figure 5: Rescheduled time space diagram

the duration of the appointment, and $f_{m}$ as the location of the appointment. Furthermore, all maintenance units still belong to their original rolling stock type (e.g. a unit of type $a$ that requires maintenance is still a unit of type a). To that end, let $b_{m} \in M$ be the corresponding rolling stock type of unit $m \in M^{\prime}$. Finally, note that rolling stock units, with a maintenance appointment at the same time, at the same location, and with the same corresponding original type, can have the same maintenance type $m \in M^{\prime}$. Then, $a_{m}$ denotes the number of units that require the specific maintenance check.

\section{Extra train unit types for maintenance}

The Extra Unit Type (EUT) model is an extension of the Composition model. By adding additional rolling stock types for every rolling stock unit that is scheduled to have a maintenance check, maintenance constraints can specifically be set on those units.

Take the same example as in the previous section. There are 25 rolling stock units, this time 10 units of type $a$ and 15 units of type $b$. There are again 2 units that require maintenance, one of type $a$ starting in Alkmaar with an appointment at Nijmegen at 16:00 and one of type $b$ starting in Den Helder with an appointment at Nijmegen at 22:00. That means that the following rolling stock types are put in the model: $a$ (9 units), $b$ (14 units), $a^{*}$ ( 1 unit) and $b^{*}$ ( 1 unit). So, two additional rolling stock types have been added to the model.

Rolling stock units that require maintenance need to be in inventory at the specified location and time for their appointment. The inventory 
is measured immediately after the coupling time, $\tau_{t}^{+}$, of every trip $t \in T$, see Constraint (3.7). Thus to be able to determine the inventory at the time of a maintenance appointment, there needs to be a trip departing from the station where the appointment takes place at exactly the time the appointment takes place. Therefore, trip $t^{\prime}$ is added to the set of trips $T$ for every maintenance appointment $m \in M^{\prime}$ with parameters $\tau_{t^{\prime}}^{d e p}=h_{m}=$ $\tau_{t^{\prime}}^{a r r}=\tau_{t^{\prime}}^{+}$and $s_{t^{\prime}}^{d e p}=f_{m}=s_{t^{\prime}}^{a r r}$. This trip is only used to measure the inventory, therefore no composition may be appointed to this trip, so the set of allowed compositions $P\left(t^{\prime}\right)$ consists of only the empty composition. To measure the inventory at the end of a maintenance appointment, we add a second artificial trip $t^{\prime \prime}$ to the set of trips $T$ for every maintenance appointment $m \in M$. This trip $t^{\prime \prime}$ has the following parameters: $\tau_{t^{\prime \prime}}^{d e p}=$ $h_{m}+g_{m}=\tau_{t^{\prime \prime}}^{a r r}$ and $s_{t^{\prime \prime}}^{d e p}=f_{m}=s_{t^{\prime \prime}}^{a r r}$. No composition may be appointed to this trip as well, so, the set of allowed compositions, $P\left(t^{\prime \prime}\right)$, consists of only the empty composition.

The units that require maintenance are added as separate rolling stock types to the set $M$, and we introduce the decision variable $A p p_{t, m}$, to denote the number of rolling stock units of type $m \in M$ that are not available at their maintenance location immediately after time $\tau_{t}^{+}$. Then, Constraint (5.1) denotes that maintenance units need to be in inventory at the time of their appointment and during the duration of their appointment. Otherwise $A p p_{t, m}$ is equal to the number of units of type $m \in M$ that are not at their appointment immediately after time $\tau_{t}^{+}$. Note that Constraint (5.1) is only for maintenance units, because the restriction $a_{m}>0$ is only true for units requiring maintenance. Furthermore, the inventory is measured immediately after every departing and arriving trip, thus a rolling stock unit cannot leave the inventory in between two measurements. As as result, a penalty value $\theta_{t}$ can be set upon units being late for their maintenance appointment, or even missing their maintenance appointment completely.

$$
\begin{array}{r}
I_{t, m}+A p p_{t, m} \geq a_{m} \quad \forall t \in T, m \in M: a_{m}>0, s_{t}^{d e p}=f_{m}, \\
h_{m} \leq \tau_{t}^{+} \leq\left(h_{m}+g_{m}\right)
\end{array}
$$

A drawback of this approach is that, by taking additional rolling stock types into account, the number of possible compositions increases rapidly. The number of possible compositions depends on the number of different rolling stock types available and on the maximum lengths of the trains. Usually no more than $5 \%$ of the rolling stock units are scheduled for maintenance during the day. Therefore, we make a simplification by restricting a composition to contain at most one maintenance unit. As a result, the increase in the number of compositions after adding an additional rolling stock type due to maintenance appointments only depends on the number of original rolling stock types. Indeed, a composition of length $k$, measured 
in the number of rolling stock units, consists of at most 1 unit that requires maintenance and at least $k-1$ original units that do not require maintenance. Denote $n$ as the number of original rolling stock types available. Adding one additional type leads thus to $n^{k-1} \cdot k$ new compositions of size $k$. The maximum length of a composition, measured in number of units, is denoted by $c$. Adding one additional rolling stock unit that requires maintenance then leads to a total increase in the number of compositions that is equal to:

$$
\begin{aligned}
& \sum_{k=1}^{c}\left(n^{k-1} \cdot k\right)=\frac{c \cdot n^{c+1}-(c+1) \cdot n^{c}+1}{(n-1)^{2}} \\
& =\frac{(c(n-1)-1) \cdot n^{c}+1}{(n-1)^{2}}
\end{aligned}
$$

This is polynomial in $n$, since $c$ is fixed, but in most cases it is not quadratic in $n$.

\section{The shadow account for maintenance}

\subsection{Introduction}

The second approach to include maintenance in the rolling stock rescheduling problem is keeping track of a shadow account (SA). A shadow account is a second rolling stock circulation with the focus on rolling stock units that require maintenance. The SA has to match with the original circulation. To that end a shadow unit is created for every available rolling stock unit. A shadow unit is not denoted by a rolling stock type (e.g. $a$ or $b$ ), but by a 'maintenance' type (e.g. 0,1,2) representing maintenance appointments. A unit with SA type 0 stands for a unit that does not require maintenance and a unit with SA type $1, \ldots, x$ stands for a unit that is scheduled to have a maintenance check. So, in the shadow account most units are of SA type 0 and just a few units have a different SA type.

For instance, consider the same situation as in the previous section. There are 10 rolling stock units of type $a$ and 15 units of type $b$. The same 2 units require maintenance, one of type $a$ starting in Alkmaar with an appointment at 16:00 in Nijmegen and one of type $b$ starting in Den Helder with an appointment at 22:00 in Nijmegen. In the normal part of the problem there are still 10 units of type $a$ and 15 units of type $b$, however in the shadow account there are 23 units of type 0 , one unit of type 1 , and one unit of type 2 .

See Figure 6 for the rolling stock circulation of all the original units. As can be seen, the original circulation represents only the original units, it is not clear which units have a maintenance appointment and which units do 
not. On the other hand, see Figure 7 for the SA circulation. In this circulation there is no distinction between units that do not require maintenance, they are all represented by light gray lines. However, there is a distinction between units that require maintenance (dark gray and black lines), so this circulation is specifically used to create maintenance paths. The two circulations have to match, otherwise the maintenance paths cannot be used.

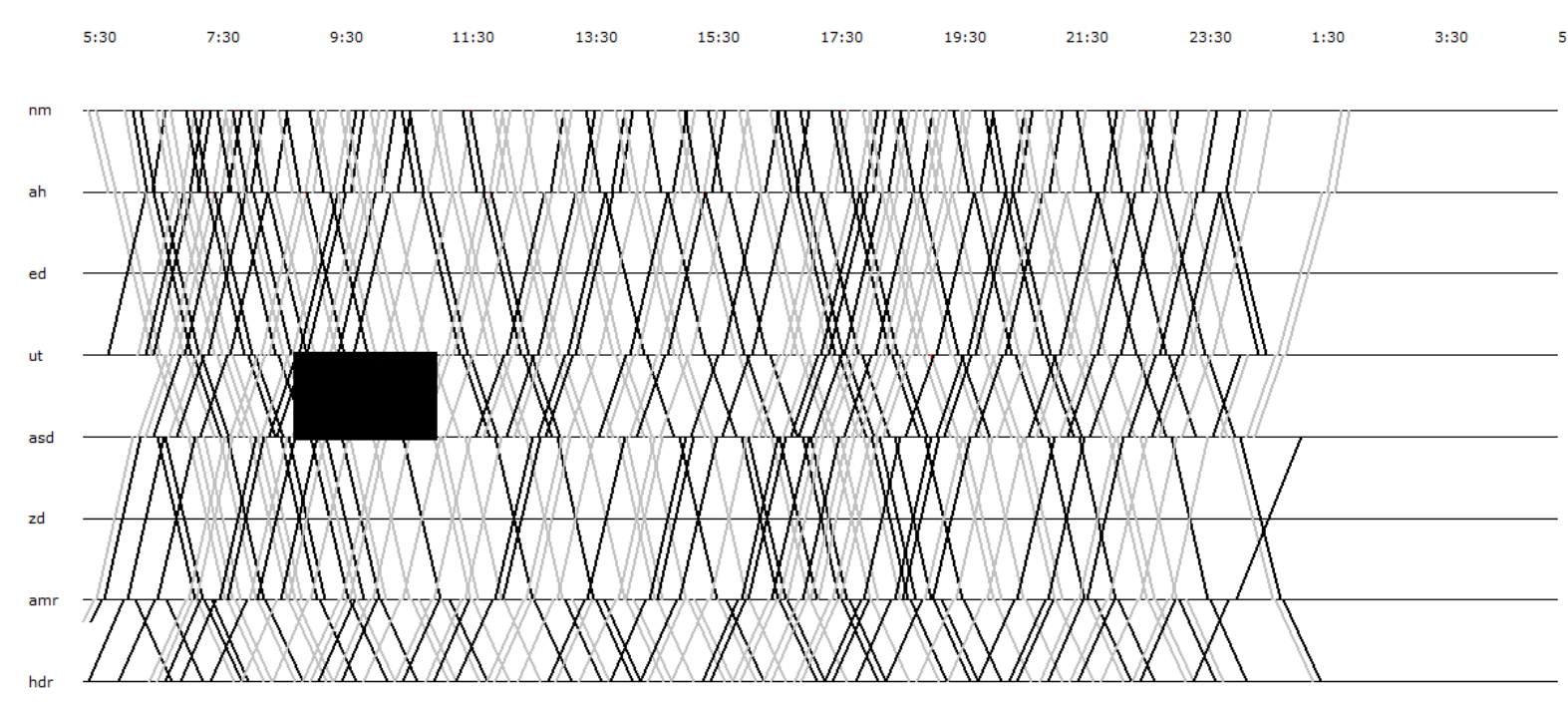

Figure 6: A solution for the Normal part, gray line $=$ type $a$, black line $=$ type $b$

As in the previous section, we denote $M$ as the set of different rolling stock types, and the maximum size of a composition is still denoted by c. Assuming that every composition contains at most one unit that requires maintenance, one can verify that the total number of additionally required compositions after adding one unit that requires maintenance equals $\sum_{k=1}^{c} k=\frac{1}{2} c(c+1)$. For example, a composition of length three (000) leads to three new compositions $(100,010,001)$. The increase is quadratic in $c$ and no longer depends on $n$.

The constraints in the complete SA model can be decomposed into three different parts: The Normal part, the SA part, and the Linking part.

The Normal part is exactly the same as the Composition model. So, the normal part consists of constraints (3.2)-(3.12). The other parts will be discussed in the coming subsections.

\subsection{Shadow Account part}

The SA part should create a rolling stock circulation for the shadow units. To that end, redefine the set $M^{\prime}$ to be the set of SA types $(0, \ldots, x)$. Let $a_{m}^{\prime}$ be 


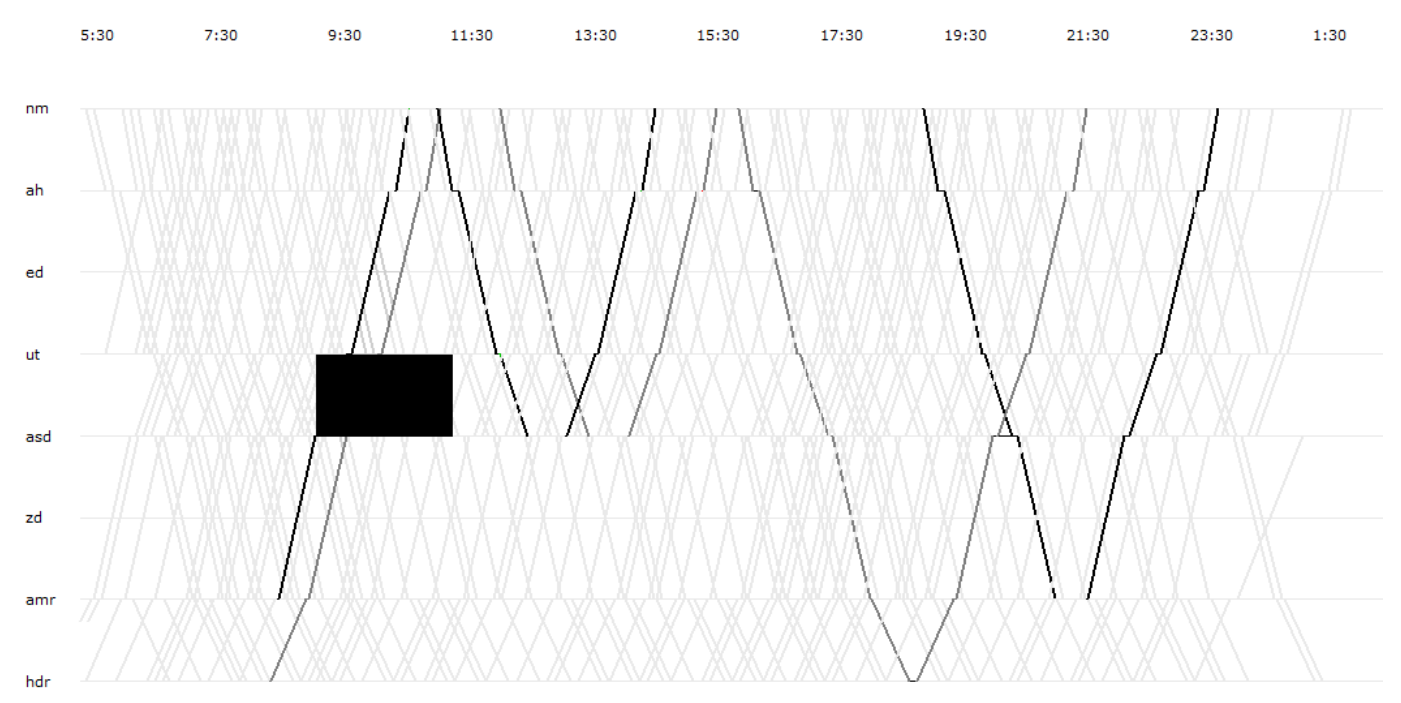

Figure 7: A matching solution for the SA part

the available number of units of type $m \in M^{\prime}$. Furthermore, $b_{m} \in M$ denotes the corresponding name of the original type of $m \in M^{\prime}$ (e.g. if a SA unit 1 corresponds to a normal unit $a$, then $b_{1}=a$ ). Let $P^{\prime}$ be the set of possible SA compositions. For each trip $t \in T, P^{\prime}(t)$ denotes the set of allowed SA compositions. Furthermore, let $Q^{\prime}$ be the set of allowed SA composition changes. Denote $\rho^{\prime}(t)$ as the allowed composition changes at the end of trip $t \in T$ in the SA part. For $q \in \rho^{\prime}(t)$ the incoming composition is denoted by $p_{q}^{\prime}$ and the outgoing composition by $o_{q}^{\prime}$. Within a given composition change $q \in Q^{\prime}, \alpha_{q, m}^{\prime}$ denotes the number of uncoupled units of type $m \in M^{\prime}$ and $\beta_{q, m}^{\prime}$ denotes the number of coupled units of type $m \in M^{\prime}$. Finally, $i_{s, m}^{\prime, 0}$ denotes the number of units of type $m \in M^{\prime}$ in the inventory at station $s \in S$ at the start of the planning period. By definition, the total number of SA units in inventory at the start of the day is equal to the number of normal units in inventory at the start of the day.

$$
\sum_{m \in M^{\prime}} i_{s, m}^{\prime, 0}=\sum_{m \in M} i_{s, m}^{0} \quad \forall s \in S
$$

Note that this is not a constraint, but a condition that is to be satisfied by the data.

The decision variables required for the SA part are:

- $X_{t, p}^{\prime} \in\{0,1\}$ denotes whether composition $p \in P^{\prime}(t)$ is used on trip $t \in T$

- $Z_{t, q}^{\prime} \in\{0,1\}$ denotes whether composition change $q \in \rho^{\prime}(t)$ is used at the end of trip $t \in T$. 
- $I_{t, m}^{\prime} \in \mathbb{Z}_{0}^{+}$denotes the number of SA units of type $m \in M^{\prime}$ in the inventory at station $s_{t}^{d e p}$ immediately after time $\tau_{t}^{+}$.

- $C_{t, m}^{\prime}$ and $U_{t, m}^{\prime} \in \mathbb{Z}_{0}^{+}$denote the number of SA units $m \in M^{\prime}$ that are coupled and uncoupled at the start and end of trip $t \in T$.

The following constraints are then used in the SA part:

$$
\begin{array}{lr}
\sum_{p \in P^{\prime}(t)} X_{t, p}^{\prime}=1 & \forall t \in T \\
X_{t, p}^{\prime}=\sum_{q \in \rho^{\prime}(t): p_{q}^{\prime}=p} Z_{t, q}^{\prime} & \forall t \in T, p \in P^{\prime}(t) \\
X_{\sigma(t), p}^{\prime}=\sum_{q \in \rho^{\prime}(t): o_{q}^{\prime}=p} Z_{t, q}^{\prime} & \forall t \in T, p \in P^{\prime}(\sigma(t)) \\
C_{\sigma(t), m}^{\prime}=\sum_{q \in \rho^{\prime}(t)} \beta_{q, m}^{\prime} Z_{t, q}^{\prime} & \forall t \in T, m \in M^{\prime} \\
U_{t, m}^{\prime}=\sum_{q \in \rho^{\prime}(t)} \alpha_{q, m}^{\prime} Z_{t, q}^{\prime} & \forall t \in T, m \in M^{\prime} \\
I_{t, m}^{\prime}=i_{s_{t}^{d e p}, m}^{\prime \prime}-\sum_{t^{\prime} \in A_{t}} C_{t^{\prime}, m}^{\prime}+\sum_{t^{\prime} \in B_{t}} U_{t^{\prime}, m}^{\prime} & \forall t \in T, m \in M^{\prime} \\
X_{t, p}^{\prime} \in\{0,1\} & \forall t \in T, p \in P^{\prime}(t) \\
C_{t, m}^{\prime}, U_{t, m}^{\prime}, I_{t, m}^{\prime} \in \mathbb{R}_{+} & \forall t \in T, m \in M^{\prime} \\
Z_{t, q}^{\prime} \in \mathbb{R}_{+} & \forall t \in T, q \in \rho^{\prime}(t)
\end{array}
$$

All SA constraints operate in the same way as the corresponding constraints in the Normal part.

\subsection{Linking part}

The Normal part creates a rolling stock circulation for the normal unit types (e.g. $a, b)$. The SA part creates a second rolling stock circulation for the SA unit types (e.g. 0,1,2). The SA part should give a shadow account of the first part. That means that the SA part should be linked to the Normal part. First of all, the solutions of both parts need to be equal in terms of the lengths of the compositions assigned to a trip, the numbers of coupled and uncoupled rolling stock units at the end of a trip, and the numbers of normal and SA rolling stock units in inventory at all times. Secondly, the flow of a rolling stock unit that requires maintenance in the SA part has to be linked to the flow of its corresponding original unit in the Normal part.

Consequently, for the first link we introduce the parameter $N_{p}$, denoting the total number of units in composition $p \in P$, and $\Upsilon$ as the set of allowed composition lengths in terms of number of units, hence $\Upsilon=\{0,1,2, \cdots, c\}$. 
Theorem 6.1. Constraint (6.11) is sufficient and necessary to connect the Normal and SA part in terms of lengths of the assigned compositions, the numbers of coupled/uncoupled units at the end of a trip, and the number of units in inventory.

$$
\sum_{p \in P(t): N_{p}=v} X_{t, p}=\sum_{p \in P^{\prime}(t): N_{p}=v} X_{t, p}^{\prime} \quad \forall t \in T, v \in \Upsilon
$$

Proof: By definition of Constraint (6.11) we have that the lengths of the compositions of each trip, expressed in the number of units in the compositions, in the Normal and SA part are equal. Furthermore, we claim that by using Equation (6.11) the variables $U_{t, m} \& U_{t, m}^{\prime}, C_{t, m} \& C_{t, m}^{\prime}$, and $I_{t, m} \& I_{t, m}^{\prime}$ are linked. We will prove this in steps.

- $U_{t, m}$ and $U_{t, m}^{\prime}$. We will show that $\sum_{m \in M} U_{t, m}=\sum_{m \in M^{\prime}} U_{t, m}^{\prime}$ for all $t \in T$ by contradiction. Assume that $\sum_{m \in M} U_{t, m}>\sum_{m \in M^{\prime}} U_{t, m}^{\prime}$ for at least one trip $t \in T$. This means that at the end of trip $t$ more units are uncoupled in the Normal than in the SA part. By definition of Constraint (6.11) the lengths of the compositions assigned to trip $t$ and its successor $\sigma(t)$ are equal. It is assumed that more units are uncoupled at the end of trip $t$ in the Normal part. This is only possible if also more units are coupled there, otherwise the lengths of the compositions assigned to trip $\sigma(t)$ are not equal. However, it is not allowed to both couple and uncouple units at the end of a trip. This leads to a contradiction, and so $\sum_{m \in M} U_{t, m} \leq \sum_{m \in M^{\prime}} U_{t, m}^{\prime}$.

The same proof holds in the other direction, thus $\sum_{m \in M^{\prime}} U_{t, m}^{\prime} \leq \sum_{m \in M} U_{t, m}$. We can conclude that

$$
\sum_{m \in M^{\prime}} U_{t, m}^{\prime}=\sum_{m \in M} U_{t, m} \quad \forall t \in T
$$

- $C_{t, m}$ and $C_{t, m}^{\prime}$, we can use the same proof as for $U_{t, m}$ and $U_{t, m}^{\prime}$ to find that:

$$
\sum_{m \in M^{\prime}} C_{t, m}^{\prime}=\sum_{m \in M} C_{t, m} \quad \forall t \in T
$$

- $I_{t, m}$ and $I_{t, m}^{\prime}$. Assume that $\sum_{m \in M} I_{t, m}>\sum_{m \in M^{\prime}} I_{t, m}^{\prime}$ immediately after time $\tau_{t}^{+}$of at least one trip $t \in T$. The inventories at the start of the day are by definition equal, see Equation (6.1), so a difference between $I_{t, m}$ and $I_{t, m}^{\prime}$ arises during the operations. Note that

$$
\sum_{m \in M} I_{t, m}=\sum_{m \in M}\left(i_{s, m}^{0}-\sum_{t^{\prime} \in A_{t}} C_{t^{\prime}, m}+\sum_{t^{\prime} \in B_{t}} U_{t^{\prime}, m}\right)
$$


and

$$
\sum_{m \in M^{\prime}} I_{t, m}^{\prime}=\sum_{m \in M^{\prime}}\left(i_{s, m}^{\prime, 0}-\sum_{t^{\prime} \in A_{t}} C_{t^{\prime}, m}^{\prime}+\sum_{t^{\prime} \in B_{t}} U_{t^{\prime}, m}^{\prime}\right)
$$

This means that a difference between $I_{t, m}$ and $I_{t, m}^{\prime}$ can only be caused by either the start inventory, $C_{t, m}$ or $U_{t, m}$, but we just showed that $\sum_{m \in M} C_{t, m}=\sum_{m \in M^{\prime}} C_{t, m}^{\prime}$ and $\sum_{m \in M} U_{t, m}=\sum_{m \in M^{\prime}} U_{t, m}^{\prime}$. So, it holds that

$$
\sum_{m \in M} I_{t, m}=\sum_{m \in M^{\prime}} I_{t, m}^{\prime} \quad \forall t \in T
$$

The second link needs to achieve that if a SA unit of type $m^{\prime} \in M^{\prime}$, with $m^{\prime} \neq 0$, is appointed to trip $t \in T$ in the SA part, then its corresponding normal type $b_{m^{\prime}} \in M$ is appointed to trip $t$ in the Normal part. To this end, denote $w_{i, p}$ as the unit assigned to position $i \in\{1, \cdots, k\}$ in composition $p \in P\left(P^{\prime}\right)$. When a SA unit of type $m^{\prime} \in M^{\prime}: m^{\prime} \neq 0$ resides in a SA composition on position $i$, then a corresponding normal unit $m \in M: m=$ $b_{m^{\prime}}$ needs to reside on position $i$ as well, see Constraint (6.12). Finally, as long as a unit of type $m^{\prime} \in M^{\prime}: m^{\prime} \neq 0$ is in inventory, a unit of type $m \in M: b_{m^{\prime}}=m$ must be in inventory as well, see Constraint (6.13).

$$
\begin{array}{rr}
\sum_{\substack{p \in P^{\prime} \\
w_{i, p}=m^{\prime}}} X_{t, p}^{\prime} \leq \sum_{\substack{p \in P \\
w_{i, p}=b_{m^{\prime}}}} X_{t, p} \quad \forall t \in T, i \in\{1, \cdots, k\}, m^{\prime} \in M^{\prime}: m^{\prime} \neq 0 \\
I_{t, m^{\prime}}^{\prime} \leq I_{t, b_{m^{\prime}}}
\end{array}
$$

In this way the SA part adheres to the Normal part and as a result constraints can be set to force a maintenance unit to be on time for its appointment. We introduce the decision variable $A p p_{t, m}$ denoting the number of rolling stock units of type $m \in M^{\prime}$ that are not in inventory at their corresponding maintenance location at time $\tau_{t}^{+}$. In the same way as in the EUT model, additional trips need to be added to the set of trips $T$ to measure the inventory at the time a maintenance appointment takes place and at the time the maintenance appointment is finished. For all rolling stock units with a maintenance appointment $m \in M^{\prime}$, we add trip $t^{\prime}$ and trip $t^{\prime \prime}$ to the set of trips $T$. The parameters of these trips are equal to: $\tau_{t^{\prime}}^{d e p}=h_{m}=\tau_{t^{\prime}}^{a r r}=\tau_{t^{\prime}}^{+}$and $s_{t^{\prime}}^{d e p}=f_{m}=s_{t^{\prime}}^{\text {arr }}$ for trip $t^{\prime}$ and $\tau_{t^{\prime \prime}}^{d e p}=h_{m}+g_{m}=\tau_{t^{\prime \prime}}^{a r r}$ and $s_{t^{\prime \prime}}^{d e p}=f_{m}=s_{t^{\prime \prime}}^{a r r}$ for trip $t^{\prime \prime}$. Because these trips are only used to measure the inventory, no composition may be appointed 
to them, so the set of allowed compositions $P\left(t^{\prime}\right), P^{\prime}\left(t^{\prime}\right), P\left(t^{\prime \prime}\right)$, and $P^{\prime}\left(t^{\prime \prime}\right)$ consists of only the empty composition.

Then, Constraint (6.14) specifies that either a maintenance unit is present at the station where its maintenance appointment is scheduled at the time of the appointment for the duration of the appointment, or the unit is too late or misses its appointment completely. The objective function is then extended with the variable $A p p_{t, m}$ and a penalty value $\theta_{t}$, just as in the EUT model. Constraint (6.14) is only needed for maintenance units $m \in M^{\prime}$, with $m \neq 0$.

$$
\begin{array}{r}
I_{t, m}^{\prime}+A p p_{t, m} \geq a_{m} \quad \forall t \in T, m \in M^{\prime}: m \neq 0, s_{t}^{d e p}=f_{m}, \\
h_{m} \leq \tau_{t}^{+} \leq h_{m}+g_{m}
\end{array}
$$

\section{Job-Composition model}

In this section the third model to take maintenance into account in the RSRP is introduced. This model is called the Job-Composition (JC) model. At the beginning of a day all rolling stock units are in inventory. During the day, a particular unit is being appointed to a certain departing trip and fulfills a number of successor trips until the particular unit is being uncoupled and becomes part of the inventory again. A job is such a sequence of successor trips between coupling and uncoupling. So, a job starts when a unit is coupled to a trip, and the job ends when the unit is uncoupled from a trip.

The problem now becomes to assign rolling stock units to both trips and jobs. In the JC model we appoint only normal units to trips, and we appoint both normal and maintenance units to jobs, while linking the units that require maintenance to their original units just as in the SA model. In this way, no additional compositions have to be taken into account for every unit that requires a maintenance appointment. A complicating factor is that certain jobs may block each other. If a job ends, it means that the unit appointed to it is uncoupled. If a different job is blocking the uncoupling, then the two jobs are called incompatible. We present constraints that prevent two incompatible jobs to be chosen. A disadvantage of this formulation is that the number of jobs can become large when there are many trips or the maximum turnaround time is long.

\section{$7.1 \quad$ Jobs}

We create a list of all possible jobs during the day and denote $J$ as this set of all possible jobs. Let $T(j)$ be the set of trips covered by job $j$. Every job $j \in J$ has a start (and final) trip denoted by $\lambda_{j}\left(\gamma_{j}\right)$. For all trips $t_{1}, \cdots, t_{n} \in T(j)$ we have that $\lambda_{j}=t_{1}, \sigma\left(t_{i}\right)=t_{i+1}$, and $t_{n}=\gamma_{j}$. As can be seen from this notation, every job $j \in J$ takes place on a route $r \in R$. Remember that the length of a predefined route depends on the shunting 
rules at a station and on the maximum turnaround time, as explained in Section 3.

Along each route $r \in R$ runs a train $v_{r}$, which consists of the actual train units that are used on the different trips within the route. Each physical train has two sides, from now on called the $A$ and $B$-side of the train. For every first trip of a route $r \in R$, we define the $A$-side to be in front. We denote $\zeta_{t}$ as the side of train $v_{r}$ that is in front at the start of trip $t \in T$ in route $r \in R$ and $\zeta_{t}^{-1}$ as the side that is in the rear.

Within a route $r \in R$, turnings can possibly take place. In, for instance, Figure 2 a route $\left(t_{1}, \cdots t_{8}\right)$ with 3 turnings is shown. After train $v_{r}$ turns, its front and rear side change. So, in order to keep track of which side is in front at the start of trip $t \in T$, we need to keep track of the number of turnings taking place in route $r(t)$ up to trip $t$. To that end, denote turn $_{t}$ as the number of turnings taking place in route $r(t)$ up to the start of trip $t \in T$. Then, $\zeta_{t}$ can be defined as in Equation (7.1).

$$
\zeta_{t}=\left\{\begin{array}{cc}
A & \text { If } \operatorname{turn}_{t} \text { is even or } 0 \\
B & \text { otherwise }
\end{array}\right.
$$

Coupling can take place at the start of trip $t \in T$. It is predefined whether a unit is coupled to the rear or to the front of the incoming train, depending on the station rules. Let $\eta_{t}$ denote whether a unit has to be coupled to the front $\left(\eta_{t}=1\right)$ or to the rear $\left(\eta_{t}=0\right)$ of the incoming train. Both the $A$ and $B$ side can be in front of the incoming train, this depends on the number of turnings taking place up to trip $t$. We define the coupling side $\omega_{j} \in\{A, B\}$ as the side where coupling takes place at the start of job $j \in J$. The coupling side is determined as in Equation (7.2).

$$
\omega_{j}= \begin{cases}\zeta_{\lambda_{j}} & \text { If } \eta_{\lambda_{j}}=1 \\ \zeta_{\lambda_{j}}^{-1} & \text { otherwise }\end{cases}
$$

At the end of trip $t$, a unit can be uncoupled from the composition. Just as with coupling, it is predefined, based on station rules, whether a unit is uncoupled from the rear or the front of the incoming train. To this end, let $\eta_{t}^{\prime}$ denote whether a unit has to be uncoupled from the front $\left(\eta_{t}^{\prime}=1\right)$ or the rear $\left(\eta_{t}^{\prime}=0\right)$ of the incoming train. This can still be the $A$ or $B$-side of the incoming train, that depends on the number of turnings taking place up to trip $t$. We denote $\pi_{j}$ as the side where uncoupling takes place at the end of job $j \in J$, called the uncoupling side. The uncoupling side is determined as in Equation (7.3).

$$
\pi_{j}= \begin{cases}\zeta_{\gamma_{j}} & \text { If } \eta_{\gamma_{j}}^{\prime}=1 \\ \zeta_{\gamma_{j}}^{-1} & \text { otherwise }\end{cases}
$$

Definition 1. A set of jobs $J^{\prime} \subset J$ on route $r \in R$ is said to be compatible, if every job $j \in J^{\prime}$, that is coupled at the start of trip $\lambda_{j} \in T$ with coupling 
side $\omega_{j}$, can be uncoupled at its uncoupling side $\pi_{j}$ after trip $\gamma_{j} \in T$ without being blocked by any other job $j^{\prime} \in J^{\prime}$.

Lemma 7.1. A set of jobs $J^{\prime} \subset J$ is compatible if and only if for each pair of jobs $j$ and $j^{\prime} \in J^{\prime}$ the following two conditions hold:

- If $\tau_{\lambda_{j}}^{d e p}<\tau_{\lambda_{j^{\prime}}}^{d e p}<\tau_{\gamma_{j}}^{a r r}<\tau_{\gamma_{j^{\prime}}}^{a r r}$, then $\omega_{j^{\prime}} \neq \pi_{j}$

- If $\tau_{\lambda_{j}^{\prime}}^{d e p}<\tau_{\lambda_{j}}^{d e p}<\tau_{\gamma_{j}}^{a r r}<\tau_{\gamma_{j^{\prime}}}^{a r r}$, then $\omega_{j}=\pi_{j}$

Proof: Assume that the set of jobs $J^{\prime}$ is compatible. Let $j$ and $j^{\prime}$ be a pair of jobs in $J^{\prime}$. First, suppose $\tau_{\lambda_{j}}^{d e p}<\tau_{\lambda_{j^{\prime}}}^{d e p}<\tau_{\gamma_{j}}^{\text {arr }}<\tau_{\gamma_{j^{\prime}}}^{\text {arr }}$. Since, by assumption, the uncoupling side of job $j$ is not blocked by job $j^{\prime}$, we have that $\omega_{j^{\prime}} \neq \pi_{j}$. Otherwise job $j$ is being blocked. Second, suppose $\tau_{\lambda_{j}^{\prime}}^{d e p}<\tau_{\lambda_{j}}^{\text {dep }}<\tau_{\gamma_{j}}^{a r r}<\tau_{\gamma_{j^{\prime}}}^{a r r}$. Again, the uncoupling of job $j$ is not blocked by job $j^{\prime}$, so we must have that $\omega_{j}=\pi_{j}$. This completes the "only-if"-part of the proof of the lemma.

Next, suppose that each pair of jobs $j$ and $j^{\prime} \in J^{\prime}$ satisfies the two condition, and that the set $J^{\prime}$ is not compatible. Then, by definition there is at least one job $j \in J$, whose uncoupling after trip $\gamma_{j}$ from its uncoupling side $\pi_{j}$ is blocked by another job $j^{\prime} \in J^{\prime}$. Clearly, $T(j) \cap T\left(j^{\prime}\right) \neq \emptyset$ and $\tau_{j}^{a r r}<\tau_{j^{\prime}}^{a r r}$. The latter follows from the fact fact that if the end times of the job are the same, then also their uncoupling sides would be the same. Thus job $j^{\prime}$ would not be blocking the uncoupling of job $j$ in that case.

Furthermore, if $\tau_{\lambda_{j}}^{d e p}=\tau_{\lambda_{j^{\prime}}}^{\text {dep }}$, then, without loss of generality, we may assume that job $j^{\prime}$ is not blocking the uncoupling of job $j$. Otherwise the positions of jobs $j$ and $j^{\prime}$ in the train could have been interchanged just before coupling. Thus we may assume that $\tau_{\lambda_{j}}^{d e p} \neq \tau_{\lambda_{j^{\prime}}}^{d e p}$. That leaves us with the cases $\tau_{\lambda_{j}}^{d e p}<\tau_{\lambda_{j^{\prime}}}^{d e p}$ and $\tau_{\lambda_{j^{\prime}}}^{d e p}<\tau_{\lambda_{j}}^{d e p}$.

If $\tau_{\lambda_{j}}^{d e p}<\tau_{\lambda_{j^{\prime}}}^{d e p}$, then we have $\tau_{\lambda_{j}}^{d e p}<\tau_{\lambda_{j^{\prime}}}^{d e p}<\tau_{\gamma_{j}}^{a r r}<\tau_{\gamma_{j^{\prime}}}^{a r r}$. Thus by assumption we have that $\omega_{j^{\prime}} \neq \pi_{j}$. In addition, if $\tau_{\lambda_{j^{\prime}}}^{d e p}<\tau_{\lambda_{j}}^{d e p}$, then we have that $\tau_{\lambda_{j^{\prime}}}^{d e p}<\tau_{\lambda_{j}}^{d e p}<\tau_{\gamma_{j}}^{a r r}<\tau_{\gamma_{j^{\prime}}}^{\text {arr }}$. Thus by assumption we have that $\omega_{j}=\pi_{j}$. However, it is clear that in both cases job $j^{\prime}$ does not block the uncoupling of job $j$. This contradiction completes the proof of the lemma.

Corollary 1. A set of jobs $J^{\prime} \subset J$ is compatible if and only if each pair of jobs in $J^{\prime}$ is compatible.

Proof: A set of jobs is compatible if each job $j \in J^{\prime}$ can be uncoupled without being blocked by another job $j^{\prime} \in J^{\prime}$. This automatically means that all jobs are pairwise compatible, which completes the "only-if" part of the proof. 
Next, suppose each pair of jobs $j, j^{\prime} \in J^{\prime}$ to be compatible and assume that set $J^{\prime}$ is not compatible. Then, by definition of an incompatible set, there must be a job $j$ whose uncoupling is blocked by a different job $j^{\prime} \in J^{\prime}$. However, that would indicate that job $j$ and $j^{\prime}$ are not compatible, which gives a contradiction and so we have that $J^{\prime}$ is compatible.

A set of jobs is not compatible if it contains a pair of jobs not fullfilling one of the two conditions in Lemma 7.1. As a result, we can add constraints to the model such that there is no such pair of jobs selected by the model. So, no pair of jobs of the sets $J P^{1}$ and $J P^{2}$, described by Equations (7.4) and (7.5), may be chosen.

$$
\begin{aligned}
& J P^{1}=\left\{\left(j, j^{\prime}\right) \in J \times J: T(j) \cap T\left(j^{\prime}\right) \neq \emptyset \wedge \tau_{\lambda_{j}}^{d e p}<\tau_{\lambda_{j^{\prime}}}^{d e p}<\tau_{\gamma_{j}}^{a r r}<\tau_{\gamma_{j^{\prime}}}^{a r r} \wedge \omega_{j^{\prime}}=\pi_{j}\right\} \\
& J P^{2}=\left\{\left(j, j^{\prime}\right) \in J \times J: T(j) \cap T\left(j^{\prime}\right) \neq \emptyset \wedge \tau_{\lambda_{j^{\prime}}}^{d e p}<\tau_{\lambda_{j}}^{d e p}<\tau_{\gamma_{j}}^{a r r}<\tau_{\gamma_{j^{\prime}}}^{a r r} \wedge \omega_{j} \neq \pi_{j}\right\}
\end{aligned}
$$

\subsection{Further notation}

During the whole day jobs are performed by rolling stock units. At the moment a disruption occurs, there are jobs already being performed by rolling stock units. Compositions of trips that have already departed at the start of the disruption cannot be changed. However, jobs can be changed, as long as the compositions appointed to the trips before the disruption takes place do not change. Denote the set of trips that have departed before the disruption and that are still operational at the start of the disruption by $T^{<} \subset T$ and set the parameter $G_{t, p}$ equal to 1 if composition $p \in P$ is assigned to trip $t \in T^{<}$.

The notation of the maintenance units is the same as in Section 4.2. We add additional trips $t^{\prime}$ and $t^{\prime \prime}$ for all rolling stock units with a maintenance appointment. These trips have the following parameters: $\tau_{t^{\prime}}^{d e p}=h_{m}=$ $\tau_{t^{\prime}}^{a r r}=\tau_{t^{\prime}}^{+}, s_{t^{\prime}}^{d e p}=f_{m}=s_{t^{\prime}}^{a r r}, \tau_{t^{\prime \prime}}^{d e p}=h_{m}+g_{m}=\tau_{t^{\prime \prime}}^{a r r}=\tau_{t^{\prime \prime}}^{+}$, and $s_{t^{\prime \prime}}^{d e p}=f_{m}=$ $s_{t^{\prime \prime}}^{a r r}$. These trips are only used to measure the inventory, so no composition may be appointed to them. Finally, the following additional variables are necessary in the JC model:

- $K_{t} \in\{0,1\}$ denotes whether trip $t \in T$ is cancelled or not.

- $W_{j} \in\{0,1\}$ denotes whether job $j \in J$ is used or not.

- $Y_{j, m} \in \mathbb{Z}^{+}$denotes the number of rolling stock units $m \in M$ that cover job $j \in J$.

- $Q_{j, m^{\prime}} \in\{0,1\}$ denotes whether job $j \in J$ is covered by a maintenance unit $m^{\prime} \in M^{\prime}$. 
- $I_{t, m^{\prime}} \in \mathbb{R}^{+}$denotes the inventory of maintenance unit $m^{\prime} \in M^{\prime}$ at station $s_{t}^{d e p}$, just after the departure of trip $t \in T$.

- $A p p_{t, m^{\prime}} \in \mathbb{Z}^{+}$denotes the number of maintenance units of type $m^{\prime} \in$ $M^{\prime}$ that are not available at their maintenance location immediately after time $\tau_{t}^{+}$.

\section{3 $\quad$ Model}

Most of the constraints of the Composition model are used in the JC model as well. The Composition model is a strong formulation with many equalities, using this part in the JC model speeds up the computation. Besides the computation time, the Composition model makes it easy to fix compositions of trips that have departed before the disruption occurs. That is why all variables and constraints of the Composition model are used in the model. 
The following additional constraints are part of the model as well:

$$
\begin{aligned}
& \min f(X, D, Z, K, A p p) \\
& \sum_{j \in J: T(j) \ni t} W_{j}+K_{t} \geq 1 \\
& \sum_{m \in M} Y_{j, m} \geq W_{j} \\
& X_{t, p}=G_{t, p} \\
& C_{t, m}=\sum_{j \in J: \lambda_{j}=t} Y_{j, m} \\
& U_{t, m}=\sum_{j \in J: \gamma_{j}=t} Y_{j, m} \\
& W_{j}+W_{j^{\prime}} \leq 1 \\
& I_{t, m^{\prime}}=i_{s_{t}^{d e p}, m^{\prime}}^{0}-\sum_{t^{\prime} \in A_{t}} \sum_{\substack{j \in J \\
\lambda_{j}=t^{\prime}}} Q_{j, m^{\prime}}+\sum_{t^{\prime} \in B_{t}} \sum_{\substack{j \in J \\
\gamma_{j}=t^{\prime}}} Q_{j, m^{\prime}} \\
& I_{t, m^{\prime}}+A p p_{t, m^{\prime}} \geq a_{m^{\prime}} \\
& Q_{j, m^{\prime}} \leq Y_{j, m} \\
& I_{t, m^{\prime}} \leq I_{t, m} \\
& W_{j} \in\{0,1\} \\
& Y_{j, m} \in \mathbb{Z}^{+} \\
& I_{t, m^{\prime}} \in \mathbb{R}^{+} \\
& Q_{j, m^{\prime}} \in\{0,1\}
\end{aligned}
$$$$
\forall j \in J
$$

$\forall t \in T^{<}, p \in P$

$\forall t \in T, m \in M$

$\forall t \in T, m \in M$

$\forall\left(j, j^{\prime}\right) \in J P^{1} \cup J P^{2}$

$\forall t \in T, m^{\prime} \in M^{\prime}$

$$
\begin{array}{r}
\forall m^{\prime} \in M^{\prime}, t \in T: s_{t}^{d e p}=f_{m^{\prime}}, \\
h_{m^{\prime}} \leq \tau_{t}^{+} \leq h_{m^{\prime}}+g_{m^{\prime}} \\
(7.14) \\
\forall j \in J, m^{\prime} \in M^{\prime}, m \in M: b_{m^{\prime}}=m \\
(7.15) \\
\forall t \in T, m^{\prime} \in M^{\prime}, m \in M: b_{m^{\prime}}=m \\
(7.16) \\
\\
(7.17) \quad \forall j \in J \\
\forall j \in J, m \in M \\
(7.18) \\
\forall t \in T, m^{\prime} \in M^{\prime} \\
(7.19) \\
\forall j \in J, m^{\prime} \in M^{\prime}
\end{array}
$$

$J$




\subsection{Explanation of the constraints}

Constraint (7.7) states that at least one job covers trip $t \in T$ or else the trip is cancelled. Every chosen job has to be performed by at least one rolling stock type $m \in M$, see Constraint (7.8). All trips $t \in T^{<}$that have departed before the start of the disruption should have the same composition as originally appointed, this is modelled by Constraint (7.9).

Constraint (7.10) states that the number of coupled units at the start of a trip is equal to the number of units that start their job at the trip, and the number of uncoupled units at the end of a trip is equal to the number of units that finish their job at the end of the trip, as modelled by Constraint (7.11). Note that Constraints (3.5) and (3.6) are required to link the Job part to the Composition part of the model, this is due to the fact that the start or end of a job in the Job part leads to a composition change in the Composition part.

At most one job of each pair of jobs in the sets $J P^{1}$ and $J P^{2}$ can be chosen to be performed. This is modelled by Constraint (7.12).

Constraint (7.13) keeps track of the inventory of maintenance units $m^{\prime} \in M^{\prime}$ at the beginning of trip $t \in T$. Constraint (7.14) states that every maintenance unit must be in inventory for the duration of its appointment, at the right location and for the right duration or else the unit was either too late or missed its appointment completely. The inventory at a station is measured at every departing trip at that station, so units cannot leave the inventory in between two trips. Just as in the SA model, linking constraints are required between the maintenance units and the corresponding normal units. If a maintenance unit $m^{\prime} \in M^{\prime}$ is used on job $j \in J$, then its corresponding original type $b_{m^{\prime}} \in M$ must also be appointed to job $j \in J$, see Constraint (7.15). The same holds for the inventory: if a maintenance unit of type $m^{\prime} \in M^{\prime}$ is in inventory, then at least one of its corresponding units of type $b_{m^{\prime}} \in M$ must also be in inventory, as is required by Constraint (7.16). Finally, Constraints (7.17)-(7.20) specify the domains of the variables.

The same objective is used as in the other models, as can be seen in Equation (7.6).

Note that the assumption that multiple maintenance units cannot occur in the same composition is no longer necessary to reduce the computation time within the JC model. This is because the number of possible composition changes does not depend on the number of maintenance units. In this paper we did not loosen this assumption, because we want to have comparable results between all three models. 


\subsection{Strengthening the formulation}

In the previously described model there are only constraints forbidding pairs of jobs to be chosen at the same time. However, these constraints can be tightened by forbidding sets, instead of pairs, of jobs to be chosen at the same time.

To that end, we define the undirected graph $G_{r}=\left(V_{r}, E_{r}\right)$, where the jobs $J$ in route $r \in R$ are the set of vertices $V_{r}$. There is an edge $e$ between every two jobs $j$ and $j^{\prime}$, if and only if $\left(j, j^{\prime}\right) \in J P_{1} \cup J P_{2}$. This means that every pair of adjacent jobs is not compatible. We call this graph the conflict graph of route $r$.

A clique is a subset of vertices $c l \subset V_{r}$, such that for every two vertices in $c l$, there exists an edge connecting the two. So, every clique of jobs, $c l \subseteq V_{r}$ within the conflict graph $G_{r}$ is a set of pairwise incompatible jobs. We could hence strengthen our formulation by adding inequalities (7.21) for some cliques $\mathrm{cl}$.

$$
\sum_{j \in c l} W_{j} \leq 1 \quad \forall r \in R, c l \subset V_{r}: c l \text { clique }
$$

Finding and adding all cliques (or all cliques which are maximal with respect to inclusion) could increase the size of the IP and the overall solution time drastically, since there could be an exponential number of those cliques. For this reason, we add only some easy to find cliques. We make use of two types of cliques, as described below.

Both types of cliques contain jobs $j^{1}, j^{2}, \ldots, j^{n} \in J$. All jobs have at least one common trip. Furthermore, for both types it holds that the unit appointed to job $j^{i}, i \in\{1, \ldots, n-1\}$, is coupled to the composition earlier than the unit appointed to job $j^{i+1}$.

The first type of cliques $\left(J B^{1}\right)$ in our conflict graph is constructed such that in each clique job $j_{1}$ is uncoupled first, then $j_{2}$ and so on. Furthermore, the uncoupling side of job $j^{i}$ is equal to the coupling side of jobs $j^{i+1}, j^{i+2}$, $\cdots, j^{n}\left(\pi_{j^{i}}=\omega_{j_{i+1}}=\omega_{j_{i+2}}=\cdots=\omega_{j^{n}}\right)$. This is not possible, because the units appointed to jobs $j^{i+1}, \cdots, j^{n}$ are in the way. So, all tuples of jobs within $J B^{1}$ are pairwise incompatible.

The second type of cliques within our conflict graph, $J B^{2}$, is defined such that in each clique job $j^{i+1}$ is uncoupled before job $j^{i}$ is uncoupled. Furthermore, the uncoupling side of job $j^{i+1}$ is different from the side where it was coupled. This is not possible, because the units appointed to job $j^{1}, j^{2}, \cdots, j^{i}$ are still there (see the set $J P^{2}$ as example of a single job being in the way). So, all tuples of jobs within $J B^{2}$ are pairwise incompatible. 


$$
\begin{aligned}
J B^{1}= & \left\{\left(j^{1}, j^{2}, \cdots, j^{n}\right) \in J \times J \times \cdots \times J: T\left(j^{1}\right) \cap T\left(j^{2}\right) \cap \cdots \cap T\left(j^{n}\right) \neq \emptyset\right. \\
& \wedge \tau_{j^{1}}^{d e p}<\tau_{j^{2}}^{d e p}<\ldots<\tau_{j^{n}}^{d e p}<\tau_{j^{1}}^{a r r}<\tau_{j^{2}}^{a r r}<\ldots<\tau_{j^{n}}^{a r r} \\
& \left.\wedge \pi_{j^{i}}=\omega_{j^{i+1}}=\omega_{j^{i+2}} \quad \forall i=1,2, \cdots, n-1\right\} \\
J B^{2}= & \left\{\left(j^{1}, j^{2}, \cdots, j^{n}\right) \in J \times J \times \cdots \times J: T\left(j^{1}\right) \cap T\left(j^{2}\right) \cap \cdots \cap T\left(j^{n}\right) \neq \emptyset\right. \\
& \wedge \tau_{j^{1}}^{d e p}<\tau_{j^{2}}^{d e p}<\ldots<\tau_{j^{n}}^{d e p}<\tau_{j^{n}}^{a r r}<\tau_{j^{n-1}}^{a r r}<\ldots<\tau_{j^{1}}^{a r r} \\
& \left.\wedge \pi_{j^{i}} \neq \omega_{j^{i}} \quad \forall i=1,2, \cdots, n\right\}
\end{aligned}
$$

There is a final constraint that can strengthen the model formulation. By definition it is not allowed to both couple and uncouple at the end of the same trip. That means that it is not allowed to start a job at the successor of the last trip of a different job. So, Constraint (7.24) can be added to the formulation as valid inequality.

$$
W_{j}+W_{j^{\prime}} \leq 1 \quad \forall\left(j, j^{\prime}\right) \in J \times J: \lambda_{j}=\sigma_{\gamma_{j^{\prime}}}
$$

\section{Results}

In this section we discuss the results of applying the EUT model, the SA model, and the JC model on a case from NS. We ran different experiments on trips of the 2200, 2800, and 3000 line in the Netherlands. Here trains are travelling from Dordrecht (Ddr) to Amsterdam (Asd) (2200 line), from Rotterdam (Rtd) to Deventer (Dv) (2800 line), and from Den Helder (Hdr) to Nijmegen (Nm) (3000 line). These lines lead to a total of 1095 trips. See Figure 8 for a visual representation.

\begin{tabular}{|c|c|c|c|}
\hline Case number & \#RS types & Turnaround time & Disrupted area \\
\hline $1 \mathrm{a}$ & 2 & 10 & $\mathrm{Gv}-\mathrm{Rtd}$ \\
\hline 1b & 2 & 10 & Ut - Asd \\
\hline $2 \mathrm{a}$ & 3 & 10 & Gv - Rtd \\
\hline $2 \mathrm{~b}$ & 3 & 10 & Ut - Asd \\
\hline $3 \mathrm{a}$ & 2 & 30 & Gv - Rtd \\
\hline $3 \mathrm{~b}$ & 2 & 30 & Ut - Asd \\
\hline $4 \mathrm{a}$ & 3 & 30 & Gv - Rtd \\
\hline $4 \mathrm{~b}$ & 3 & 30 & Ut - Asd \\
\hline
\end{tabular}

Table 1: Different cases

Table 1 gives an overview of the cases on which the models are tested. Here, "\#RS" types denotes the number of original rolling stock types used. 


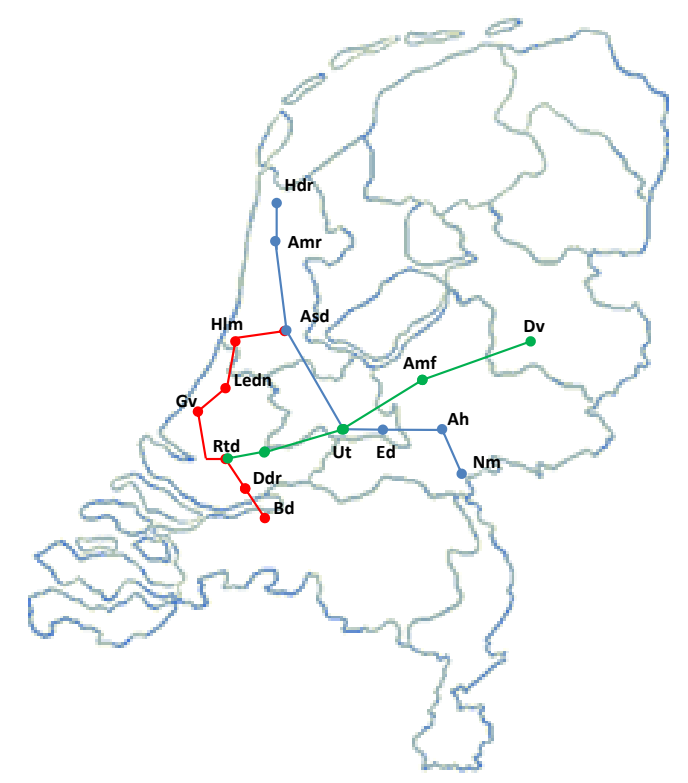

Figure 8: Case lines

As can be seen, this is either two or three. In the cases with two rolling stock types, the rolling stock units consist of either three or four carriages, while in the cases with three different rolling stock types they consist of either three, four or five carriages. The maximum number of carriages in a train equals 15 , so in total there are 31 compositions and 356 composition changes possible when using two different rolling stock types, and 72 compositions and 884 composition changes are possible when using three different rolling stock types.

The column "Turnaround time" defines the maximum time a train is allowed to wait for its successor trip. As explained in Section 3, trips within a predefined route have a successor trip. We call the amount of time between two succeeding trips, when turning takes place, the turnaround time. There is a maximum amount of time allowed between two succeeding trips, called the maximum turnaround time. The length of a route depends on the maximum turnaround time at the end stations in the route. If a rolling stock unit is allowed to wait a long time at a track before departing on its successor trip, then there are many successor trips. So, with a long maximum turnaround time, routes can become longer. The turnaround time is fixed by the input data. For instance, with a turnaround time of 10 minutes, a train scheduled to wait more than ten minutes at a platform is transported 
to the shunting area, instead of waiting for its successor trip to depart. As a result, the trip does not have a successor trip, since the three lines are operated twice per hour in both directions. On the other hand, in the cases with a turnaround time of 30 minutes most of the trips will have a successor trip. This leads to an increase in the number of possible jobs in the JC model, which influences the computation times.

In the column "Disrupted area" the location where the disruption takes place is given. A disruption takes either place between the stations The Hague (Gv) and Rotterdam (Rtd) or between the stations Utrecht (Ut) and Amsterdam (Asd). In order to test whether the start time of the disruption has any influence on the computation time, we let disruptions take place between 07:00-09:00, 07:03-09:03, 07:06-09:06, ..., and 07:57 - 09:57, so in total 20 different time slots. Furthermore, we experiment with a number of rolling stock units requiring maintenance between one and six. All of the cases in Table 1 are solved for the different time slots and the different numbers of units requiring maintenance. As a result, there are in total $8 * 20 * 6=960$ cases per model.

\begin{tabular}{|c|c|}
\hline Parameter & Penalty \\
\hline Cancelling & 10000 \\
\hline EOD deviation & 100 \\
\hline Capacity shortage kilometer & 1 \\
\hline Carriage kilometer & 1 \\
\hline Deviation original plan & 50 \\
\hline Missing maintenance & 300 \\
\hline
\end{tabular}

Table 2: Objective function values

Table 2 gives an overview of the objective function coefficients used to solve the problem instances. Here, "Cancelling" denotes the penalty for cancelling a trip; "EOD deviation" means the penalty for deviating from the scheduled end of day balance; "Capacity shortage" kilometer stands for the penalty on the number of passengers that do not fit in an appointed train measured per kilometer. Note that we do not take changing passenger demand into account. All operated trains are assumed to have unchanged passenger demand. Taking accurate passenger demand into account is outside our scope, see Kroon et al. [7] for a paper that does take it into account. "Carriage kilometer" is the penalty on the number of carriages appointed per kilometer; "Deviation original plan" means the penalty on the difference between the original and rescheduled plan in numbers of couplings and uncouplings taking place; "Missing maintenance" stands for the penalty on the number of rolling stock units that miss their scheduled maintenance appointment.

All computations are ran with CPLEX 12.5.1 and an Intel (R) Core 
(ITM) i5-3210M processor with $2.50 \mathrm{GHz}$ and 8GB RAM. The maximum computation time is set to 500 seconds per case and the allowed gap size is set to $0 \%$.

An important note to make before presenting the results, is that all models give the same optimal solution, in the cases where they were able to prove optimality, which was in most of the cases. Therefore, we will compare the models in terms of computation speed and number of found optimal solutions.

\subsection{Results turnaround time 10 minutes}

We start with showing the results when the maximal turnaround time equals 10 minutes.

First, the results of using two normal types are shown in Table 3a, Table $3 \mathrm{~b}$, and Figure 9. As can be seen, the JC model performs significantly better than the SA and the EUT model: both the computation times, and the numbers of found optimal solutions are better in the JC model than for the EUT and SA model. Furthermore, the EUT model is not able to find an optimal solution within 500 seconds in some of the cases when there are many units requiring maintenance.

\begin{tabular}{|l|r|r|r|r|r|}
\hline I & Model & Time & \#NF & \#C & \#V \\
\hline \multirow{4}{*}{1} & EUT & 58 & 0 & 165339 & 1063531 \\
& JC & 38 & 0 & 83992 & 421309 \\
& SA & 64 & 0 & 132646 & 645108 \\
\hline \multirow{4}{*}{2} & EUT & 95 & 0 & 274219 & 1823416 \\
& JC & 66 & 0 & 87004 & 438030 \\
& SA & 87 & 0 & 168126 & 855405 \\
\hline \multirow{4}{*}{3} & EUT & 133 & 0 & 383100 & 2562576 \\
& JC & 74 & 0 & 90017 & 439832 \\
& SA & 110 & 0 & 203607 & 1065572 \\
4 & EUT & 172 & 0 & 474373 & 3216358 \\
& JC & 83 & 0 & 92309 & 441043 \\
& SA & 161 & 0 & 229304 & 1205559 \\
\hline \multirow{5}{*}{5} & EUT & 268 & 0 & 568219 & 393142 \\
& JC & 97 & 0 & 94641 & 452064 \\
& SA & 213 & 0 & 260132 & 1443211 \\
\hline \multirow{4}{*}{6} & EUT & 380 & 6 & 666201 & 4803231 \\
& JC & 112 & 0 & 97032 & 459216 \\
& SA & 256 & 1 & 291240 & 1603491 \\
\hline
\end{tabular}

(a) Case $1 \mathrm{a}$

\begin{tabular}{|r|r|r|r|r|r|}
\hline $\mathrm{I}$ & Model & Time & \#NF & $\# \mathrm{C}$ & $\# \mathrm{~V}$ \\
\hline \multirow{4}{*}{1} & EUT & 51 & 0 & 165444 & 1064512 \\
& $\mathrm{JC}$ & 50 & 0 & 84115 & 436677 \\
& $\mathrm{SA}$ & 57 & 0 & 132762 & 645703 \\
\hline \multirow{4}{*}{2} & EUT & 97 & 0 & 274498 & 1825098 \\
& $\mathrm{JC}$ & 60 & 0 & 87104 & 438490 \\
& $\mathrm{SA}$ & 106 & 0 & 168275 & 856194 \\
\hline \multirow{4}{*}{3} & EUT & 138 & 0 & 383490 & 2585684 \\
& $\mathrm{JC}$ & 92 & 0 & 90166 & 440303 \\
& $\mathrm{SA}$ & 128 & 0 & 203789 & 1066685 \\
\hline \multirow{4}{*}{4} & EUT & 227 & 0 & 474856 & 3219325 \\
& $\mathrm{JC}$ & 76 & 0 & 92461 & 441531 \\
& $\mathrm{SA}$ & 199 & 0 & 229509 & 1206651 \\
\hline \multirow{4}{*}{5} & EUT & 328 & 2 & 569021 & 394261 \\
& JC & 105 & 0 & 96721 & 452497 \\
& SA & 239 & 0 & 260405 & 1443981 \\
\hline \multirow{3}{*}{6} & EUT & 417 & 8 & 667091 & 4805197 \\
& JC & 118 & 0 & 97333 & 460134 \\
& SA & 279 & 1 & 291865 & 2604754 \\
\hline
\end{tabular}

(b) Case $1 b$

Table 3: Results with 2 normal types and 10 minutes turnaround time 


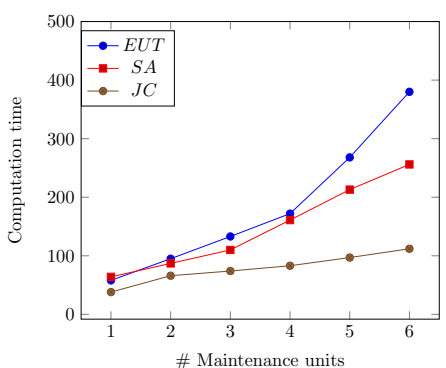

(a) Ut-Asd

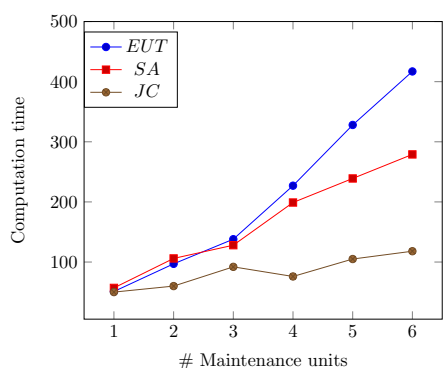

(b) Rtd-Gv

Figure 9: Computation times with 2 normal types and 10 minutes turnaround time

The results of applying the models on instances with three normal types are shown in Table 4a, Table $4 \mathrm{~b}$ and Figure 10. The JC model performs again best, both in terms of computation speed and the number of found optimal solutions. The SA model is second and the EUT model performs worst, having difficulty to solve cases with many maintenance appointments.

The JC model performs better, because it does not need any additional compositions for an additional unit that requires maintenance. The more additional rolling stock units requiring maintenance, the more beneficial this becomes. As can be seen, it results in less variables and constraints than the SA and EUT model require.

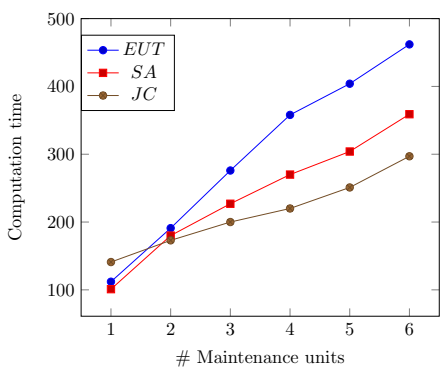

(a) Ut-Asd

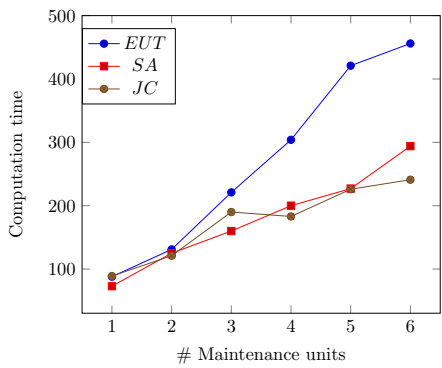

(b) Rtd-Gv

Figure 10: Computation times with 3 normal types and 10 minutes turnaround time

\subsection{Results with turnaround time 30 minutes}

In contrast with the results with a turnaround time of 10 minutes, the SA model outperforms both the EUT and the JC model when the turnaround time equals 30 minutes. The JC model now has many more jobs, which makes it harder to solve. The JC model has difficulties to find a solution from the start, but the number of rolling stock units requiring maintenance 


\begin{tabular}{|r|r|r|r|r|r|r|r|r|r|r|r|}
\hline I & Model & Time & \#NF & \#C & \#V & I & Model & Time & \#NF & \#C & $\# \mathrm{~V}$ \\
\hline \multirow{4}{*}{1} & EUT & 112 & 0 & 305545 & 2048938 & & EUT & 88 & 0 & 305856 & 2050828 \\
& JC & 141 & 0 & 169642 & 1013684 & 1 & JC & 89 & 0 & 169852 & 1014675 \\
& SA & 101 & 0 & 219016 & 1221847 & & SA & 73 & 0 & 219217 & 1222974 \\
\hline \multirow{4}{*}{2} & EUT & 191 & 0 & 471149 & 32174916 & & EUT & 131 & 0 & 471629 & 3220459 \\
& JC & 173 & 0 & 172654 & 1015486 & 2 & JC & 121 & 0 & 172877 & 1016488 \\
& SA & 180 & 0 & 257384 & 1432144 & & SA & 125 & 0 & 257618 & 1433465 \\
\hline \multirow{4}{*}{3} & EUT & 276 & 5 & 572205 & 3921008 & & EUT & 221 & 1 & 572788 & 3924625 \\
& JC & 200 & 0 & 175666 & 1017288 & 3 & JC & 190 & 0 & 175902 & 1018301 \\
& SA & 227 & 0 & 285972 & 1571981 & & SA & 160 & 0 & 286229 & 1573431 \\
\hline \multirow{4}{*}{4} & EUT & 358 & 10 & 737810 & 5089561 & & EUT & 304 & 3 & 738562 & 5094256 \\
& JC & 220 & 0 & 178679 & 1019090 & 4 & JC & 183 & 0 & 178928 & 1020114 \\
& SA & 270 & 0 & 324341 & 1782278 & & SA & 200 & 0 & 324631 & 1783922 \\
\hline \multirow{4}{*}{5} & EUT & 404 & 14 & 885807 & 6131286 & & EUT & 421 & 11 & 886710 & 6136942 \\
& JC & 251 & 0 & 181688 & 1020892 & 5 & JC & 226 & 0 & 181950 & 1021927 \\
& SA & 304 & 2 & 352926 & 1922115 & & SA & 227 & 0 & 353239 & 1923888 \\
\hline \multirow{2}{*}{6} & EUT & 462 & 16 & 986867 & 6834803 & & EUT & 456 & 15 & 987873 & 6841108 \\
& JC & 297 & 1 & 184704 & 1022694 & 6 & JC & 241 & 0 & 184979 & 1023740 \\
& SA & 359 & 4 & 381618 & 2061952 & & SA & 294 & 2 & 381854 & 2063854 \\
\hline
\end{tabular}

(a) Case $2 \mathrm{a}$

(b) Case $2 \mathrm{~b}$

Table 4: Results with 3 normal types and 10 minutes turnaround time

does not influence the computation time that much.

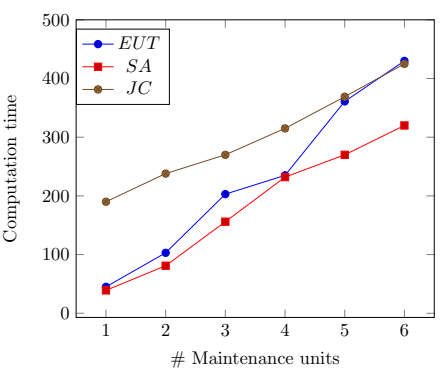

(a) Ut-Asd

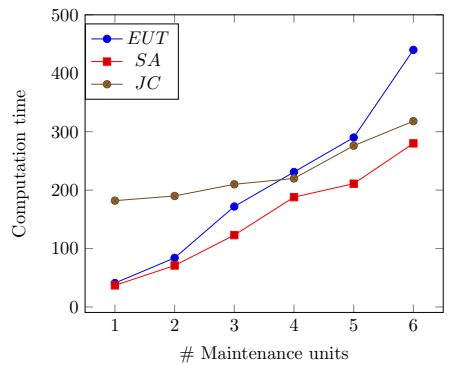

(b) Rtd-Gv

Figure 11: Computation times with 2 normal types and 30 minutes turnaround time

In the case with 2 rolling stock types, the results are shown in Tables 5a and $5 \mathrm{~b}$ and Figure 11. As can be seen, all models tend to be slower than in the case with a turnaround time of 10 minutes.

When comparing the EUT and SA model, it can be seen that the SA model outperforms the EUT model, both in terms of computation times and in terms of the numbers of found optimal solutions. This is due to the fact 


\begin{tabular}{|l|r|r|r|r|r|}
\hline I & Model & Time & \#NF & $\# \mathrm{C}$ & $\# \mathrm{~V}$ \\
\hline \multirow{4}{*}{1} & EUT & 45 & 0 & 175221 & 1063525 \\
& $\mathrm{JC}$ & 190 & 0 & 148931 & 443767 \\
& $\mathrm{SA}$ & 39 & 0 & 142730 & 645102 \\
\hline \multirow{4}{*}{2} & EUT & 103 & 0 & 291243 & 1823410 \\
& $\mathrm{JC}$ & 238 & 0 & 153389 & 447078 \\
& $\mathrm{SA}$ & 81 & 0 & 183456 & 855399 \\
\hline \multirow{4}{*}{3} & EUT & 203 & 0 & 407249 & 2583295 \\
& $\mathrm{JC}$ & 270 & 0 & 157845 & 450389 \\
& $\mathrm{SA}$ & 156 & 0 & 224180 & 1065696 \\
\hline \multirow{4}{*}{4} & EUT & 235 & 0 & 504462 & 3216352 \\
& $\mathrm{JC}$ & 315 & 1 & 162300 & 453700 \\
& $\mathrm{SA}$ & 232 & 0 & 252641 & 1205533 \\
\hline \multirow{4}{*}{5} & EUT & 361 & 2 & 602319 & 3904671 \\
& JC & 369 & 3 & 167027 & 456918 \\
& SA & 270 & 0 & 280120 & 1360012 \\
\hline \multirow{4}{*}{6} & EUT & 430 & 7 & 700232 & 4700129 \\
& JC & 425 & 8 & 171872 & 499812 \\
& SA & 320 & 2 & 301321 & 1421208 \\
\hline
\end{tabular}

(a) Case $3 \mathrm{a}$

\begin{tabular}{|r|r|r|r|r|r|}
\hline I & Model & Time & \#NF & $\# \mathrm{C}$ & $\# \mathrm{~V}$ \\
\hline \multirow{4}{*}{1} & EUT & 41 & 0 & 175340 & 1064506 \\
& JC & 182 & 0 & 137231 & 443456 \\
& SA & 37 & 0 & 141586 & 645697 \\
\hline \multirow{4}{*}{2} & EUT & 84 & 0 & 291522 & 1825092 \\
& JC & 190 & 0 & 141613 & 446626 \\
& SA & 71 & 0 & 181967 & 856188 \\
\hline \multirow{4}{*}{3} & EUT & 172 & 0 & 407639 & 2585678 \\
& JC & 210 & 0 & 145993 & 449796 \\
& SA & 123 & 0 & 222346 & 1066679 \\
\hline \multirow{4}{*}{4} & EUT & 231 & 0 & 504882 & 3219319 \\
& JC & 220 & 0 & 150372 & 452966 \\
& SA & 188 & 0 & 253262 & 1206645 \\
\hline \multirow{4}{*}{5} & EUT & 290 & 2 & 607123 & 3924021 \\
& JC & 296 & 1 & 154871 & 456723 \\
& SA & 211 & 1 & 282432 & 1399864 \\
\hline \multirow{3}{*}{6} & EUT & 410 & 7 & 710023 & 4647910 \\
& JC & 398 & 5 & 158120 & 459102 \\
& SA & 280 & 1 & 310212 & 15812904 \\
\hline
\end{tabular}

(b) Case 3b

Table 5: Results with 2 normal types and 30 minutes turnaround time

that the SA model requires less additional compositions for every additional unit requiring maintenance.

The JC model is slower than with a turnaround time of 10 minutes. As explained before this is due to the fact that the number of possible jobs increased. On the other hand, the computation time for the JC model does not increase as quickly as for the other models when more maintenance units are required. In the end, it performs even better than the EUT model when 6 rolling stock units have a maintenance appointment.

In conclusion, the SA model performs better than both the JC and the EUT model when using two normal types and a turnaround time of 30 minutes.

The results when using three different original types are shown in Tables $6 \mathrm{a}, 6 \mathrm{~b}$ and Figure 12. As can be seen, the SA model outperforms both the JC and the EUT model in terms of computation times and number of found optimal solutions again.

The JC model takes longer to find an optimal solution from the start, but the computation time does not increase quickly when more rolling stock units require maintenance. Only in the case with six maintenance units the JC model is not able to find an optimal solution for any of the cases. Note that the model was able to find feasible solutions for most cases with 


\begin{tabular}{|r|r|r|r|r|r|r|r|r|r|r|r|}
\hline I & Model & Time & \#NF & \#C & \#V & I & Model & Time & \#NF & \#C & $\# \mathrm{~V}$ \\
\hline \multirow{4}{*}{1} & EUT & 104 & 0 & 324633 & 2049838 & & EUT & 75 & 0 & 324628 & 2048938 \\
& JC & 366 & 3 & 252781 & 1026777 & 1 & JC & 387 & 4 & 235246 & 1023441 \\
& SA & 116 & 0 & 232064 & 1221847 & & SA & 152 & 0 & 232229 & 1221847 \\
\hline \multirow{4}{*}{2} & EUT & 152 & 0 & 482393 & 3069888 & & EUT & 166 & 0 & 482867 & 3093514 \\
& JC & 410 & 9 & 257343 & 1030192 & 2 & JC & 388 & 5 & 239729 & 1026715 \\
& SA & 151 & 0 & 257159 & 1361684 & & SA & 179 & 0 & 257335 & 1361684 \\
\hline \multirow{4}{*}{3} & EUT & 194 & 0 & 590055 & 3794180 & & EUT & 281 & 4 & 590112 & 3794180 \\
& JC & 430 & 10 & 258106 & 1030312 & 3 & JC & 420 & 7 & 244215 & 1029989 \\
& SA & 180 & 0 & 282257 & 1501521 & & SA & 240 & 1 & 282444 & 1501521 \\
\hline \multirow{4}{*}{4} & EUT & 262 & 4 & 747896 & 4835905 & & EUT & 300 & 8 & 747882 & 4835905 \\
& JC & 460 & 12 & 262743 & 1033742 & 4 & JC & 440 & 10 & 248698 & 1033263 \\
& SA & 210 & 1 & 307352 & 1641358 & & SA & 260 & 3 & 307550 & 1641358 \\
\hline \multirow{4}{*}{5} & EUT & 341 & 9 & 855553 & 5508888 & & EUT & 363 & 11 & 855538 & 5539422 \\
& JC & 470 & 15 & 267380 & 10377172 & 5 & JC & 460 & 11 & 253181 & 1036537 \\
& SA & 222 & 2 & 332447 & 1781195 & & SA & 290 & 4 & 332656 & 1781195 \\
\hline \multirow{2}{*}{6} & EUT & 427 & 14 & 963208 & 6242939 & & EUT & 482 & 14 & 963196 & 6242939 \\
& JC & - & 20 & 272003 & 1040602 & 6 & JC & - & 20 & 257664 & 1039811 \\
& SA & 241 & 2 & 357582 & 1921032 & & SA & 340 & 8 & 357782 & 1921032 \\
\hline
\end{tabular}

(a) Case $4 \mathrm{a}$

(b) Case $4 \mathrm{~b}$

Table 6: Results with 3 normal types and 30 minutes turnaround time

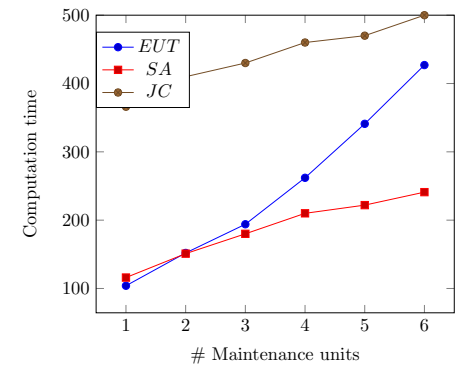

(a) Ut-Asd

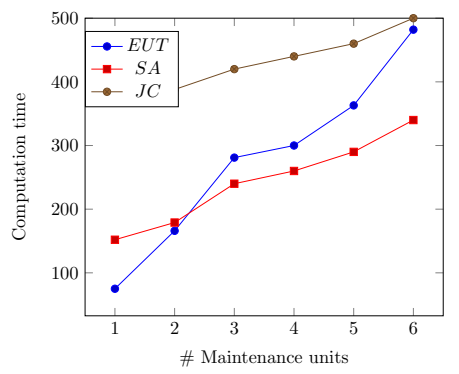

(b) Rtd-Gv

Figure 12: Computation times with 3 normal types and 30 minutes turnaround time

6 maintenance units, however it was not able to prove optimality within the time window of 500 seconds.

To conclude, the SA model outperforms both the JC and the EUT model in terms of computation time and number of optimal solutions found when there are three normal types and a turnaround time of 30 minutes. 


\section{Conclusions and further research}

In this paper, three new models are presented for rescheduling the rolling stock of passenger trains during large disruptions, while taking scheduled maintenance appointments into account.

The first model is the EUT model, which is an extension of the already existing Composition model. By adding additional types for every rolling stock unit that requires maintenance, constraints can be set on them. This extension has a drawback that adding additional types leads to a rapid increase in the number of possible compositions and composition changes. As a result, the EUT model tends to require more computation time when more units require maintenance.

The second model is the SA model. Within the SA model a shadow account for all rolling stock units is kept. In this way, maintenance constraints can easily be put on the rolling stock units that require maintenance. The results show that the SA model performs better than the EUT model and better than the JC model with a long turnaround time.

The third and final model is the JC model. This model assigns rolling stock units to jobs. As a result, paths are created for every rolling stock unit. Specific paths can be created for rolling stock units that require maintenance. The JC model performs best on the tested instances with a turnaround time of 10 minutes.

There are several directions for further research. Firstly, a column generation technique could be used to create paths for individual rolling stock units. In this way maintenance constraints can be set upon the units that require maintenance. The main problem for using a column generation technique is that the order of units in compositions are of importance, and, as a result, the columns are highly dependent on each other. This is in contrast with, for instance, crew rescheduling where the different crew members per train are independent of each other. Secondly, other practical aspects are of importance to be included in the RSRP. For instance, station routing, robustness, and accurate passenger demand forecasting during disruptions. Finally, an integrated approach to tackle both timetable rescheduling, rolling stock rescheduling and crew rescheduling is desirable in future research.

\section{References}

[1] R. Ahuja, T. Magnanti, and J. Orlin. Network Flows: Theory, Algorithms and Applications. Prentice-Hall Englewood Cliffs, 1993.

[2] C. Barnhart, N.L. Boland, L.W. Clarke, E. Johnson, G.L. Nemhauser, and R.G. Shenoi. Flight string models for aircraft fleeting and routing. Transportation Science, 32(3):208-220, 1998. 
[3] R. Borndörfer, M. Reuther, T. Schlechte, and S. Weider. A hypergraph model for railway vehicle rotation planning. Proc. 11th Workshop on Algorithmic Approaches for Transportation Modelling, Optimization, and Systems (ATMOS 2011), 20:11-36, 2011.

[4] C. Cacchiani, D. Huisman, M. Kidd, L.G. Kroon, P. Toth, L. Veelenturf, and J. Wagenaar. An overview of recovery models and algorithms for real-time railway rescheduling. Transportation Research Part B: Methodological, 63:15-37, 2014.

[5] L. Clarke, E. Johnson, and G. Nemhauser. The aircraft rotation problem. Annals of Operations Research, 69:139-151, 1997.

[6] P. Fioole, L.G. Kroon, G. Maróti, and A. Schrijver. A rolling stock circulation model for combining and splitting of passenger trains. European Journal of Operational Research, 174:1281-1297, 2006.

[7] L.G. Kroon, G. Maróti, and L.K. Nielsen. Rescheduling of railway rolling stock with dynamic passenger flows. Technical Report, No. ERS2010-045-LIS, Erasmus Research Institute of Management, Rotterdam, 2010. Forthcoming in Transportation Science.

[8] G. Maróti. Operations research models for railway rolling stock planning. PhD thesis, Eindhoven University of Technology, The Netherlands, 2006.

[9] G. Maróti and L.G. Kroon. Maintenance routing for train units: the transition model. Transportation Science, 39(4):518-525, 2005.

[10] G. Maróti and L.G. Kroon. Maintenance routing for train units: the interchange model. Computers \& Operations Research, 34(4):1121-1140, 2007.

[11] L.K. Nielsen. Rolling stock rescheduling in passenger railways. Ph.D thesis, Erasmus University, Rotterdam, The Netherlands, 2011.

[12] L.K. Nielsen, L.G. Kroon, and G. Maróti. A rolling horizon approach for disruption management of railway rolling stock. European Journal of Operational Research, 220:496-509, 2012.

[13] K. Sato and N. Fukumura. Real-time freight locomotive rescheduling and uncovered train detection during disruptions. European Journal of Operational Research, 221:636-648, 2012.

[14] K. Sato, S. Sakikawa, T. Morita, N. Ueki, and T. Murata. Crew and vehicle rescheduling based on a network flow model and its application to a railway train operation. IAENG International Journal of Applied Mathematics, 39(3), 2009. 
[15] K.T. Talluri. The four day aircraft maintenance routing problem. Transportation Science, 32(1):43-53, 1998. 


\begin{tabular}{|c|c|}
\hline \multicolumn{2}{|c|}{ ERIM Report Series Research in Management } \\
\hline ERIM Report Series reference number & ERS-2015-002-LIS \\
\hline Date of publication & $2015-02-18$ \\
\hline Version & $18-02-2015$ \\
\hline Number of pages & 38 \\
\hline Persistent URL for paper & http://hdl.handle.net/1765/77585 \\
\hline Email address corresponding author & jwagenaar@rsm.nl \\
\hline Address & $\begin{array}{l}\text { Erasmus Research Institute of Management } \\
\text { (ERIM) } \\
\text { RSM Erasmus University / Erasmus School } \\
\text { of Economics } \\
\text { Erasmus University Rotterdam } \\
\text { PO Box } 1738 \\
3000 \text { DR Rotterdam, The Netherlands } \\
\text { Phone: +31104081182 } \\
\text { Fax: +31104089640 } \\
\text { Email: info@erim.eur.nl } \\
\text { Internet: http://www.erim.eur.nl }\end{array}$ \\
\hline Availability & $\begin{array}{l}\text { The ERIM Report Series is distributed } \\
\text { through the following platforms: } \\
\text { RePub, the EUR institutional repository } \\
\text { Social Science Research Network (SSRN) } \\
\text { Research Papers in Economics (RePEc) }\end{array}$ \\
\hline Classifications & $\begin{array}{l}\text { The electronic versions of the papers in the } \\
\text { ERIM Report Series contain bibliographic } \\
\text { metadata from the following classification } \\
\text { systems: } \\
\text { Library of Congress Classification (LCC) } \\
\text { Journal of Economic Literature (JEL) } \\
\text { ACM Computing Classification System } \\
\text { Inspec Classification Scheme (ICS) }\end{array}$ \\
\hline
\end{tabular}

